\title{
Construction of Convergent Simplicial Approximations of Quantum Fields on Riemannian Manifolds
}

\author{
Sergio Albeverio $\star \star \star$ and Boguslav Zegarlinski \\ Fakultät für Mathematik, Ruhr-Universität D-4630 Bochum, Federal, Republic of Germany and SFB \\ 237-Bochum-Essen-Düsseldorf
}

\section{Dedicated to Res Jost and Arthur Wightman}

\begin{abstract}
We construct simplicial approximations of random fields on Riemannian manifolds of dimension $d$. We prove convergence of the fields to the continuum limit, for arbitrary $d$ in the Gaussian case and for $d=2$ in the non-Gaussian case. In particular we obtain convergence of the simplicial approximation to the continuum limit for quantum fields on Riemannian manifolds with exponential interaction.
\end{abstract}

\section{Introduction}

Quantum fields on Riemannian manifolds have become recently a topic of major interest in several connections. Besides the well known open problem of formulating a mathematical theory of quantum gravity, in which quantum fields on manifolds are traditionally thought to play a fundamental role, see e.g. [As], [HaI], we might mention the explosion of activity in the study of quantum strings (and superstrings), in which conformal fields over two dimensional Riemannian manifolds play a central role, see e.g. [GSW], [AHK PS1,2]. More generally the study of conformal fields with their relations to representation theory of infinite dimensional algebras and groups (see e.g. [Kac], [PrS], [FreLM], [AHKMTT]), statistical mechanics of 2-dimensional lattice systems (see e.g. [Kau], [ISZ]), completely integrable systems (see e.g. [ISZ]) and topological objects like knots and braids (see e.g. [ISZ], [Frö], [RehS], [Ga]) has attracted in recent years great interest, both in mathematics and physics. We also mention that the theory of geometrical fields like gauge fields, Markov cosurfaces, Higgs fields (see e.g. [BaJ], [Gr2], [GrKS], [AHKH], [AHKHK1,2,3], [AHKI], [AIK]) also can be looked at as a natural extension of the theory of scalar quantum fields on Riemannian manifolds.

Also from the point of view of stochastic analysis there is interest in studying random processes and fields on Riemannian manifolds. To quote a couple of contexts where this interest arises let us mention the role played by properties of

$\star$ BiBoS Research Centre

$\star \star$ CERFIM (Locarno) 
diffusion processes (and random walks) in the study of differential geometrical structures (and groups theoretical also), see e.g. [El], [ElR], the role of random fields in stochastic geometry (integral geometry), see e.g. [Sa], and in the modelling of rough or fractal structures, see e.g. [Ma], [Ad]. The interest in extending stochastic analysis from flat space to manifolds and from the study of scalar functions to the one of differential forms has also been pointed out from different points of view, e.g. [I], [WoZ].

For constructing and studying quantum and random fields on manifolds a useful tool is a simplicial approximation. In the case where the manifold is $\mathbb{R}^{d}$ discrete regular approximations (replacing $\mathbb{R}^{d}$ by $\delta \mathbb{Z}^{d}$ ) have been already studied in detail. Let us mention classical work starting with Wiener and culminating with Donsker's invariance theorem (and extensions thereof) (see e.g. [Wi]), as far as processes are concerned, or the lattice approximation of Euclidean random and quantum fields, see e.g. [GRS], [Si], [GlJ], [AFHKL]. In the latter case convergence has been proven for two and three space-time dimensional models. Also for gauge fields and stochastic connections regular lattice approximations have been studied and shown to converge in certain cases to the continuum limit, see e.g. [CDeA], [Gr], [Dr], [AHKH], [DeADFG].

Simplicial approximations have appeared in the physics and mathematical literature in connection with quantum gravity, see e.g. [Re], [CMS], [Kha], string theory [AmD] and gauge fields models, see e.g. [DrM]. However to our knowledge convergence to the continuum limit has not been proven in any model.

In the present paper we study systematically the simplicial approximation of random and quantum fields on manifolds.

We exploit some basic results achieved in the mathematical literature concerning finite element methods, e.g. [StrF], [Nit], and important work on approximations of eigenvalues and differential forms on Riemannian manifolds done by Dodziuk, Dodziuk-Patodi and Müller in connection with the proof of the Ray-Singer conjecture on equivalence of analytic and combinatorial torsion.

In Sect. 1 we recall basic concepts of algebraic topology like complexes and triangulations (Sect. 1.1) and introduce combinatorial differential operators as basic elements of simplicial approximation theory for differential operators or forms (Sect. 1.2) (as developed in [Do], [DoP], [Mü], [Eck]).

In Sect. 2 we introduce real-valued simplicial fields associated with $q$-cochains over a complex of a smooth triangulation of a (compact) connected Riemannian manifold. We also define the analogue of Euclidean free quantum field measures for simplicial fields and prove their convergence to the continuum limit as the mesh of the triangulation converges to zero.

In Sect. 3 we study (correlation) inequalities for real-valued simplicial fields on a manifold. We prove certain inequalities of Brascamp-Lieb type for Gaussian measures associated with simplicial fields with free respectively Neumann boundary conditions. We also provide an example to illustrate that the relation between fields with Dirichlet boundary conditions and fields with free or Neumann boundary conditions does not hold, in general, for triangulations of Riemannian manifolds. We discuss both the cases with mass term and without it. In particular we prove that locally we can bound expectations of the 0 -simplicial measures 
associated with a Riemannian manifold with arbitrary (non-degenerate) metric by expectations with a measure corresponding to free Euclidean field scaled by a finite factor.

In Sect. 4 we introduce local interactions for simplicial fields on compact 2-dimensional Riemannian manifolds. In fact we give two possible definitions of Wick powers and exponential functions of the free simplicial fields. We show their belonging to the relevant $L^{p}$ respectively $L^{2}$ spaces (here we also use a result in [FröZe1]).

In Sect. 5 we prove that the simplicial approximations of Wick powers and exponential functions introduced in Sect. 4 converge to a well defined continuum limit as the mesh of the approximations goes to zero. We also provide a representation of simplicial Wick powers fields in the same probability space as the free Euclidean fields.

In Sect. 6 we study the simplicial approximation of a model of scalar random and quantum fields on a 2-dimensional Riemannian manifold with exponential interaction. This model has been studied originally for the case of $\mathbb{R}^{2}$ (HøeghKrohn's model), see [AHK]. For its relevance for (bosonic) string theory (Liouville's model) see [AHKPS1], [AHKPS2]. See also [AFHKL], [AHPRS], [AR1,2], [A], $[\mathrm{Sc}],[\mathrm{AK}],[\mathrm{Ku}]$ for further references and work on Høegh-Krohn's model. In this paper we prove bounds of Brascamp-Lieb type for the interacting simplicial fields with exponential interaction and their continuum limit. We also point out some consequences of these inequalities and of the convergence result.

Let us finally remark that we expect our ideas to be also useful for providing a rigorous foundation for topological quantum field theory.

\section{Preliminaries}

1.1 Complexes and Triangulations. In this section we shall introduce some basic concepts of algebraic topology, referring to [SiTh], [Whi], e.g., as basic references. Let $V$ be a vector space over $\mathbb{R}$. Let $k \in \mathbb{N} \cup\{0\}$. Let $\left\{v_{0}, \ldots, v_{k}\right\}$ be vectors in $V$ such that $\left\{v_{1}-v_{0}, v_{2}-v_{0}, \ldots, v_{k}-v_{0}\right\}$ is linear independent. The convex set generated by $\left(v_{0}, \ldots, v_{k}\right\}$ is denoted by $\left[v_{0}, v_{1}, \ldots, v_{k}\right]$ and called a (closed) $k$-simplex (in $\mathbb{R}^{k}$ ). The set $\left\{v \in\left[v_{0}, v_{1}, \ldots, v_{k}\right], v=\sum_{i=0}^{k} a_{i}^{(v)} v_{i}, a_{i}^{(v)}>0, i=0, \ldots, k\right\}$ is called an open $k$-simplex and denoted by $\left(v_{0}, \ldots, v_{k}\right)$. The vertices of $\left[v_{0}, \ldots, v_{k}\right]$ are the points $v_{0}, \ldots, v_{k}$. The closed faces of $\left[v_{0}, \ldots, v_{k}\right]$ are the closed simplices $\left[v_{j_{0}}, v_{j_{1}}, \ldots, v_{j_{h}}\right]$, where $\left\{j_{0}, \ldots, j_{h}\right\}$ is a nonempty subset of $\{0,1, \ldots, k\}$. The open faces of $\left[v_{0}, \ldots, v_{k}\right]$ are the open simplices $\left(v_{j_{0}}, \ldots, v_{j_{k}}\right)$.

A simplical (Euclidean) complex $K$ is a finite set of open simplices $\sigma_{i}^{(q)}$, in some $\mathbb{R}^{D}$, where $i$ runs over a finite subset $I_{K}$ of $\mathbb{N}, \sigma_{i}^{(q)}$ is an open $q$-simplex, $0 \leqq q \leqq D$, such that

i) if $\sigma_{i}^{(q)} \in K$ then all open faces of its closure belong to $K$,

ii) for any $0 \leqq q \leqq D$, if $\sigma_{i}^{(q)} \cap \sigma_{j}^{(q)} \neq \varnothing$, then $\sigma_{i}^{(q)}=\sigma_{j}^{(q)}$.

One calls the maximum dimension of the simplexes of $K$ the dimension of $K$. Let $[K]=\bigcup_{q, j} \sigma_{j}^{(q)}$, then $[K]$ is compact. 
For any zero simplex $\sigma_{i}^{(0)} \equiv\left[p_{i}\right], p_{i} \in \mathbb{R}^{D}$, i.e. vertex, its star is defined as the open set

$$
S t\left(p_{i}\right) \equiv\left\{\bigcup_{j} \sigma_{j}^{(q)} \mid p_{i} \in \overline{\sigma_{j}^{(q)}}, \sigma_{j}^{(q)} \in K\right\} .
$$

For any $q$-simplex $\sigma^{(q)}$ its star is by definition, the union of all simplexes $\sigma^{\left(q^{\prime}\right)} \in K$, $q^{\prime} \geqq q$ such that $\sigma^{(q)} \subset \sigma^{\left(q^{\prime}\right)}$ (i.e. the union of all open simplices of $K$ such that $\sigma^{(q)}$ is a face of $\left.\sigma^{\left(q^{\prime}\right)}\right)$. Now let $\bar{\sigma}^{(q)}$ be a $q$-simplex, with vertices $v_{0}, \ldots, v_{q}$. Two orderings $\left(v_{j_{1}}, \ldots, v_{j_{q}}\right)$ and $\left(v_{k_{1}}, \ldots, v_{k_{q}}\right)$ of the vertices of $\bar{\sigma}^{(q)}$ are equivalent if $\left(k_{1}, \ldots, k_{q}\right)$ is an even permutation of $\left(j_{1}, \ldots, j_{q}\right)$, (this defines indeed an equivalence relation). An oriented simplex $\bar{\sigma}^{(q)}$ is a simplex together with a choice of one of the equivalence classes. If $v_{0}, \ldots, v_{q}$ are the vertices of $\tilde{\sigma}^{(q)}$, the oriented simplex determined by the ordering $\left(v_{0}, \ldots, v_{q}\right)$ will be denoted by $\left\langle v_{0}, \ldots, v_{q}\right\rangle$ (this is the same as orienting the $q$-plane containing $\bar{\sigma}^{(q)}$ by means of the ordered basis $\left\{v_{1}-v_{0}, \ldots, v_{q}-v_{0}\right\}$.

Let $K$ be a simplicial complex. Let $C_{q}(K, \mathbb{R}) \equiv C_{q}(K)$ be the set of all formal linear combinations, chains:

$$
\sigma \equiv \sum_{i} a_{i}\left\langle\sigma_{i}\right\rangle, \quad a_{i} \in \mathbb{R}, \sigma_{i}=\left(v_{0}, \ldots, v_{q}\right) \in K
$$

subject to the identifications $-a_{i}\left\langle v_{0}, \ldots, v_{q}\right\rangle=a_{i}\left\langle v_{1}, v_{0}, \ldots, v_{q}\right\rangle$.

For simplicity in the following we often do not differentiate between $\left\langle\sigma_{i}\right\rangle$ and $\sigma_{i}$ and write simply $\sum_{i} a_{i} \sigma_{i}$ for a chain. $C_{q}(K)$ is a vector space (over $\mathbb{R}$ ) of dimension the number of $q$-simplices of $K$. We define the star of the chain $\sigma$ as the union of $s t \sigma_{i}$ for the $i$ for which $a_{i} \neq 0$. Let $\langle s\rangle=\left\langle v_{0}, \ldots, v_{q+1}\right\rangle$ be an oriented $(q+1)$-simplex. The boundary $\partial\langle s\rangle$ of $\langle s\rangle$ is the $q$-chain defined by

$$
\partial\langle s\rangle \equiv \sum_{j=0}^{q+1}(-1)^{j}\left\langle v_{0}, v_{1}, \ldots, \hat{v}_{j}, \ldots, v_{q+1}\right\rangle,
$$

where ^over a symbol means that symbol is deleted.

The boundary map $C_{q}(K) \stackrel{\partial}{\longleftarrow} C_{q+1}(K)$ is a group homomorphism defined by

$$
\partial\left(\sum a_{i}\left\langle\sigma_{i}\right\rangle\right)=\sum a_{i} \partial\left\langle\sigma_{i}\right\rangle
$$

One has easily $\partial^{2}=\partial \cdot \partial=0$. For $0 \leqq q \leqq \operatorname{dim} K$, let $C^{q}(K) \equiv C_{q}(K)^{*}$, where $*$ denotes the dual of $C_{q}(K)$ (linear maps from $C_{q}(K)$ into $\mathbb{R}$ ) defined by the canonical pairing $\langle$,$\rangle on K$ defined by

$$
\left\langle\sigma_{i}^{(q)}, \sigma_{j}^{\left(q^{\prime}\right)}\right\rangle=\delta^{q q^{\prime}} \delta_{i j}, \quad \sigma_{i}^{(q)}, \sigma_{j}^{\left(q^{\prime}\right)} \in K .
$$

We call $C^{q}(K)$ the space of $q$-cochains (it can of course be identified with $C_{q}(K)$ ). Every cochain $\varphi^{(q)} \in C^{q}(K)$ can then be written as

$$
\varphi^{(q)}=\sum_{j} \varphi_{j}^{(q)}\left\langle\sigma_{j}^{(q)}\right\rangle
$$

with $\varphi_{j}^{(q)} \in \mathbb{R}$, the summation being over all (oriented) $q$-simplexes in $K$. Let $d^{c} \equiv \partial^{*}: C^{q}(K) \rightarrow C^{q+1}(K)$ be the adjoint of the boundary map

$$
C_{q+1}(K) \stackrel{\partial}{\rightarrow} C_{q}(K)
$$


Thus $d^{c}$ is defined by

$$
\left[d^{c}(\varphi)\right](c)=\varphi(\partial c)
$$

with

$$
\varphi \in C^{q}(K), \quad c \in C_{q+1}(K)
$$

One has easily $d^{c} \cdot d^{c}=0$.

Let $C(K)$ be the union of all $C^{q}(k), 0 \leqq q \leqq D$. We can look upon $d^{c}$ as a map from $C(K)$, into itself. $d^{c}$ is called the coboundary operator.

For each oriented $q$-simplex $\langle\sigma\rangle$ of $K$, let $\varphi_{\langle\sigma\rangle} \in C^{q}(K)$ be defined by

$$
\varphi_{\langle\sigma\rangle}\left\langle\sigma^{\prime}\right\rangle \equiv\left\{\begin{array}{rll}
1 & \text { if } & \left\langle\sigma^{\prime}\right\rangle=\langle\sigma\rangle \\
-1 & \text { if } & \left\langle\sigma^{\prime}\right\rangle=-\langle\sigma\rangle \\
0 & \text { if } & \sigma^{\prime} \neq \sigma .
\end{array}\right.
$$

So if $\left\langle\sigma_{1}\right\rangle, \ldots,\left\langle\sigma_{m}\right\rangle$ is a basis for $C_{q}(K)$, then $\varphi_{\left\langle\sigma_{1}\right\rangle}, \ldots, \varphi_{\left\langle\sigma_{m}\right\rangle}$ is the dual basis of $C^{q}(K)$.

We have then

$$
d^{c} \varphi_{\left\langle v_{0}, \ldots, v_{q}\right\rangle}=\sum_{v}^{\prime} \varphi_{\left\langle v, v_{0}, \ldots, v_{q}\right\rangle}
$$

where $\sum_{v}^{\prime}$ denotes the sum over all vertices $v \in K$ such that $\left(v, v_{0}, \ldots, v_{q}\right)$ is a $(q+1)$-simplex of $K$.

Let $M$ be a (compact, connected and oriented) smooth (Riemannian) manifold of dimension $D$. Let $\partial M$ be its boundary (which can be void).

Let $h:([K],[L]) \rightarrow(M, \partial M)$ be a smooth triangulation of $M$, i.e. $K$ and $L$ are simplicial complexes, $L \subset K, h$ is an homeomorphism such that for each complex of $K$, the map $h:[\sigma] \rightarrow M$ has an extension $h_{\sigma}$ to an open neighborhood $U$ of $[\sigma]$ in the plane of $[\sigma]$ such that $h_{\sigma}: U \rightarrow M$ is a smooth submanifold; and correspondingly with $K$ replaced by $L$ and $M$ by $\partial M$.

Now let $K$ be any simplicial complex, with vertexes $p_{1}, \ldots, p_{m}$. Suppose $p \in[K]$. For $j \in\{1, \ldots, m\}$, the $j^{\text {th }}$ barycentric coordinate $b_{p_{j}}(p)$ of $p$ is defined as follows. If $p \notin \operatorname{St}\left(p_{j}\right)$, then $b_{p_{j}}(p)=0$; if $p \in \operatorname{St}\left(p_{j}\right)$, then $p \in(\sigma)$ for some simplex $\sigma$ having $p_{j}$ as a vertex, and $b_{p_{j}}(p)$ is equal to the barycentric coordinate of $p$ in $\sigma$ relative to the vertex $p_{j}$ in the sense that $p=\sum_{j=1}^{m} b_{p_{j}}(p) p_{j}$. One has $b_{p_{j}}$ in $[K] \rightarrow \mathbb{R}$ continuous, $b_{p_{J}}(p) \geqq 0, \sum_{j=1} b_{p_{J}}(p)=1$ for each $p \in[K]$. Identifying $[K]$ with $M$ by the existence of the above homeomorphism $h$ we can look upon the $b_{p_{j}}, p$ as points in $M$ and call $b_{p_{1}}$ the barycentric coordinates of the point $p$ in $M$.

1.2 Combinatorial Differential Operators. In this section we present some results of simplicial approximation theory for differential operators or forms developed in [Do], [DoP], [Mü] (see also [StrF]). Let us first introduce the continuum version of the basic object we shall consider. Let $M$ be a smooth manifold. Let $T(M, p)$ respectively $T^{*}(M, p)$, for $p \in M$, be the tangent respectively cotangent space at $p$ and let $\Lambda^{k}\left(T^{*}(M, p)\right)$ be the space of all anti-symmetric $k$-linear functions on 
$T(M, p)$, called $k$-forms associated with $T^{*}(M, p)$. Let $\Lambda^{k} \equiv \Lambda^{k}(M) \equiv \bigcup_{p \in M} \Lambda^{k}\left(T^{*}(M, p)\right)$. Let $\pi$ be the projection map from $\Lambda^{k}(M)$ onto $M$. Let $\alpha$ be a mapping for $M$ into $\Lambda^{k}(M)$ such that $\pi \cdot \alpha=i_{M}$ (the identity on $M$ ). $\alpha$ is called a $k$-form on $M$. A $k$-form $\alpha$ is called smooth or $C^{\infty}$ if $\alpha\left(v_{1}, \ldots, v_{k}\right) \in C^{\infty}(M, \mathbb{R})$, where $v_{i}$ are smooth vector fields on $M$ and $\alpha\left(v_{1}, \ldots, v_{k}\right)(p) \equiv \alpha(p)\left(v_{1}(p), \ldots, v_{k}(p)\right)$. A differential form on $M$ is a mapping $\alpha: \underset{D}{\rightarrow} G(M)$ such that $\pi \cdot \alpha=i_{M}$, where $G(M) \equiv \bigcup_{p \in M} G\left(T^{*}(M, p)\right)$, with $G\left(T^{*}(M, p)\right) \equiv \bigoplus_{k=0}^{D} \Lambda^{k}\left(T^{*}(M, p)\right)$, with $D \equiv$ dimension $M$, the exterior (Grassmann) algebra of $T^{*}(M, p)$. A differential form is smooth if its components in $\Lambda^{k}(M)$ are smooth for each $k$. The set of smooth $k$-forms on $M$ is denoted by $C^{\infty}\left(M, \Lambda^{k}(M)\right)$ or for simplicity by $\Lambda^{k}$ alone. $C^{\infty}(M, \Lambda(M))$, or for simplicity $\Lambda$, denotes the set of all differential forms. Let a metric $g$ be given on $M$. Let $*$ be the Hodge star operator defined on $\Lambda$ (by $\left.{ }^{*} \beta \wedge \alpha=\# \alpha\right\lrcorner \beta$, with $\beta \in C^{\infty}\left(M, \Lambda^{k}(M)\right), \alpha \in C^{\infty}\left(M, \Lambda^{k}(M)\right.$ ), with $\# \alpha$ the tangent $k$ vector corresponding to $\alpha$. $\lrcorner$ is contraction defined by

$$
\begin{aligned}
\bigcup_{p} T_{p}(M) \times \Lambda^{k}(M) & \rightarrow \Lambda^{k-1}(M), \\
(a, \alpha) & \rightarrow a\lrcorner \alpha, \\
a\lrcorner \alpha\left(a_{2}, a_{3}, \ldots, a_{k}\right) & \equiv \alpha\left(a, a_{2}, \ldots, a_{k}\right) .
\end{aligned}
$$

A natural scalar product in $\Lambda$ is defined by

$$
(\alpha, \beta) \equiv \int_{M} \alpha \wedge * \beta,
$$

for $\alpha, \beta \in \Lambda^{k}(q=1, \ldots, D)$. Let $d: \Lambda \rightarrow M$ denote the exterior derivative (exterior differentiation operator, antiderivation) of degree +1 , uniquely defined (see e.g. [Wa]) by $d^{2}=0$ and $d f$ the differential of $f$ if $f \in C^{\infty}(M)$.

Let $L^{2} \Lambda$ be the closure of $\Lambda$ in the norm defined by the scalar product (1.12). For $\partial M=\emptyset$, let $\delta$ be the adjoint of $d$ with respect to the scalar product (1.12). Define as Laplace-Beltrami operator on $M$ the operator $\Delta$ given by

$$
-\Delta \equiv \overline{(d+\delta)^{2} \uparrow \Lambda}
$$

the bar meaning operator closure (and $\uparrow$ meaning restriction to $\Lambda$ ) (closure exists by symmetry).

If $\partial M \neq \varnothing$ we shall have additionally to consider the boundary conditions. Let $\Lambda_{D} \subset \Lambda$ be the subset consisting of (smooth) differential forms vanishing at the vicinity of $\partial M$. The Laplace-Beltrami operator with Dirichlet (or relative) boundary conditions on $\partial M$ is given by

$$
-\Delta_{D} \equiv \overline{(d+\delta)^{2} \uparrow \Lambda_{D}}
$$

Similarly, for $\Lambda_{N} \subset \Lambda$ a subset of differential forms, with vanishing their normal part and normal derivative on the boundary, we define the Laplace-Beltrami operator with Neumann (or absolute) boundary conditions on $\partial M$.

$$
-\Delta_{N} \equiv \overline{(d+\delta)^{2}\left\lceil\Lambda_{N}\right.} .
$$


If $\partial M$ consists of disconnected components one can also consider other (self-adjoint) boundary conditions.

Now let $M$ be a compact, connected and oriented. For any Laplace-Beltrami operator $\Delta$ defined above, let $-\lambda_{n}^{(q)}$, and $\phi_{n}^{(q)}$ denote its eigenvalues and respectively corresponding eigenforms in $\Lambda^{q}$, i.e.

$$
-\Delta \phi_{n}^{(q)}=\lambda_{n}^{(q)} \phi_{n}^{(q)} .
$$

It is known that, $M$ being a compact smooth manifold, $-\Delta$ has a discrete nonnegative spectrum. For any $0 \leqq q \leqq D$ we can and do order the eigenvalues so that $\lambda_{n}^{(q)} \leqq \lambda_{n+1}^{(q)}$. The Green operator is by definition the map $G: L^{2} \Lambda \rightarrow L^{2} \Lambda$ satisfying for any $\alpha \in \Lambda^{q}, G \alpha \in \Lambda^{q}$ and moreover

$$
-\Delta G \alpha=\alpha-H \alpha
$$

where $H$ denotes the projection in $L^{2} \Lambda$ on the subspace of harmonic forms (i.e. forms $\alpha$ satisfying $\Delta \alpha=0$ ).

In the approximation theory the important role is played by the Whitney mapping defined as follows [Whi]:

Definition 1.1. Let $K$ be a smooth triangulation of $M$, with vertices $p_{0}, \ldots, p_{n}$. The Whitney mapping is a linear function

$$
W: C(K) \rightarrow L^{2} \Lambda
$$

defined as follows: For $\left[p_{i}\right] \in C^{0}(K)$

$$
W\left[p_{i}\right]:=b_{p_{i}},
$$

where $b_{p_{i}}$ is the barycentric coordinate associated to $p_{i}$. For

$$
1 \leqq q \leqq D
$$

and

$$
\sigma^{(q)} \equiv\left(p_{0}, p_{1}, \ldots, p_{q}\right) \in C^{q}(K)
$$

we set

$$
W \sigma^{(q)}:=q ! \sum_{i=0}^{q}(-1)^{i} b_{p_{i}} d b_{p_{0}} \wedge \cdots \wedge d b_{p_{i-1}} \wedge d b_{p_{i+1}} \wedge \cdots \wedge d b_{p_{q}} .
$$

The fact that our triangulation is smooth implies that each continuous function $b_{p_{i}}$ is smooth outside the $(D-1)$-dimensional set $\partial \overline{S t\left[p_{i}\right]}$ (where here-means closure and $\partial$ boundary). Moreover for any $\sigma^{(q)}$ we can apply the exterior derivative $d$ to $W \sigma^{(q)}$ and the resulting form $d W \sigma^{(q)}$ is in $L^{2} \Lambda^{(q+1)}$. For a cochain $\sigma \in C^{q}(K)$, let $W \sigma$ be the Whitney form associated to $\sigma$ via the map given in Definition 1.1. One can easily show, see [Do], that the Whitney map is local in the sense that

$$
W \sigma=0 \quad \text { on } \quad M \backslash \overline{S t \sigma}
$$

and has the property

$$
W d^{c} \sigma=d W \sigma
$$


Following [Do], ([Ec]), we define the following scalar product in the space of cochains:

Definition 1.2 For $\sigma \in C^{q}(K), \sigma^{\prime} \in C^{q^{\prime}}(K)$ we define

$$
\left(\sigma, \sigma^{\prime}\right):=\left(W \sigma, W \sigma^{\prime}\right) \equiv \int W \sigma \wedge^{*} W \sigma^{\prime}
$$

if $q=q^{\prime}$ and zero otherwise.

As pointed out in [Eck] (see also [Do], [DoP] [Mü]) any scalar product in $C(K)$ leads to a combinatorial Hodge theory. Using the scalar product (1.21) we define an operator $\delta^{c}$ formal adjoint to $d^{c}$, as an operator satisfying

$$
\left(\delta^{c} \sigma, \sigma^{\prime}\right)=\left(\sigma, d^{c} \sigma^{\prime}\right)
$$

for some $\sigma \in C(K)$ and all $\sigma^{\prime} \in C(K)$. If $\partial M$ is empty (1.22) uniquely determines $\delta^{c}$. In this case we define a combinatorial Laplacian by

$$
-\Delta^{c} \equiv\left(d^{c}+\delta^{c}\right)^{2} .
$$

If the boundary $\partial M$ is nonempty we can supplement (1.22) by suitable boundary conditions.

Restricting ourselves to a subset $C_{0}(K)$ consisting of chains $c$ vanishing on the boundary, i.e. $W c=0$ on $\partial M$, we get the operators $d_{D}^{c}$ and its adjoint $\delta_{D}^{c}$. Using them we define combinatorial Laplacian with Dirichlet (relative) boundary conditions on $\partial M$ by

$$
-\Delta_{D}^{c}:=\left(d_{D}^{c}+\delta_{D}^{c}\right)^{2} .
$$

For $\partial M \neq \varnothing$ and if no restriction on the domain is taken, we define the combinatorial Laplacian with Neumann (absolute) boundary conditions on $\partial M$ by (1.23) and denote it by $\Delta_{N}^{c}$.

Additionally, for the case of nonempty and nonconnected boundary one can also consider combinatorial Laplacians with mixed boundary conditions. If it will be clear which combinatorial Laplacian we have in mind or some properties will be satisfied for all boundary conditions, we will simply use $\Delta^{c}$ to denote any combinatorial Laplacian under consideration.

Consider a combinatorial Laplacian $\Delta^{c}$ restricted to a space of $q$-chains for some $0 \leqq q \leqq D$. Let $\phi_{l}^{c} \in C^{q}(K)$ be its normalized eigenvector corresponding to an eigenvalue $-\lambda_{l}^{c}$, i.e.

$$
-\Delta^{c} \phi_{l}^{c}=\lambda_{l}^{c} \phi_{l}^{c}
$$

and $\left(\phi_{l}^{c}, \phi_{l}^{c}\right)=1$, (the scalar product being defined in (1.21)) with indexing by $l \in \mathbb{N}$ so that

$$
\lambda_{l}^{c} \leqq \lambda_{l+1}^{c} .
$$

By our definition, $0 \leqq \lambda_{l}^{c}$ and in particular for Dirichlet boundary conditions $0<\lambda_{i}^{c}$. Let $H^{(q)}$ denote a subspace of $C^{q}(K)$ consisting of harmonic $q$-cochains i.e. $\phi^{c} \in H^{(q)}$ if

$$
-\Delta^{c} \phi^{c}=0 .
$$

By $H$ we will denote also the projection operator onto the subspace of harmonic cochains. Let $G^{c}: C(K) \rightarrow C(K)$ be the combinatorial Green operator corresponding to $\Delta^{c}$ i.e. a unique linear map satisfying: 
for any $\alpha \in C^{q}(K), G^{c} \alpha$ is a $q$-cochain being in the domain of $\Delta^{c}$ and

$$
-\Delta^{c} G^{c} \alpha=\alpha-H \alpha
$$

Definition 1.4. The De Rham map is a function

$$
R: \Lambda \rightarrow C(K)
$$

satisfying for any $0 \leqq q \leqq D$

$$
\Lambda^{q} \ni \alpha \mapsto R \alpha:=\sum\left(\int_{\sigma} \alpha\right) \sigma,
$$

where the summation is over $\sigma, \sigma$ running over all $q$-simplexes in $K$ (identified by use of the canonical pairing (1.5) with all $q$-cosimplexes in $\left.C^{q}(K)\right) \cdot \int_{\sigma} \alpha$ is the integral
of $\alpha$ over $\sigma$.

We remark that de Rham map is well defined also on the subset $W R \Lambda$ of $L^{2} \Lambda$ and we have the following property [Do]:

$$
R W \sigma=\sigma \text {. }
$$

For any $\alpha \in \Lambda$, the form $W R \alpha$ can be considered as an approximation of $\alpha$. In this section we would like to describe the behaviour of this approximation, as well as the behaviour of eigenvalues $\lambda^{c}$ and eigenforms $\phi^{c}$ of the combinatorial Laplacian $\Delta^{c}$, when a complex $K$ becomes "dense." For this we will consider a well behaved family of smooth triangulations $\left\{K_{n}\right\}_{n \in \mathbb{N}}$ satisfying $K_{n} \subset K_{n+1}$, i.e. $K_{n+1}$ is a subdivision of $K_{n}$.

To characterise more precisely such a family we introduce the following two quantities: A mesh $\eta$ of the smooth triangulation $K$ is by definition a number

$$
\eta \equiv \eta(K)=\sup _{\sigma \in K} \operatorname{diam}(\sigma) \text {. }
$$

A fullness $\Theta$ of $K$ is by definition a number

$$
\Theta \equiv \Theta(K)=\inf \frac{\mathrm{Vol} \sigma}{\eta^{D}}
$$

with infimum taken over all $D$-simplexes, $D \equiv \operatorname{dim} M$, in $K$.

Given $K$ one can construct a family $\left\{K_{n}\right\}_{n \in \mathbf{N}}$ by making successively standard subdivisions of $K$ as follows [Whi] ([Do]):

Let $\sigma=\left(p_{0}, p_{1}, \ldots, p_{q}\right)$ be a simplex in $\mathbb{R}^{D}, q \leqq D$. The vertices of $S \sigma$, the standard subdivision of $\sigma$, are by definition the points

$$
p_{i j}=\frac{1}{2}\left(p_{i}+p_{j}\right), \quad i \leqq j .
$$

Now we define a partial ordering of the vertices of $S \sigma$. Following [Whi] one can choose it as follows:

$$
p_{i j} \leqq p_{k l} \quad \text { if } \quad i \geqq k \quad \text { and } \quad j \leqq l
$$

and declare that the simplexes of $S \sigma$ are all those formed from $p_{i j}$ which are in increasing order. (In this way for a $q$-simplex $\sigma$ we get $2^{q}$ simplexes in $S \sigma$.) For a complex $K$ we define its standard subdivision $S K$ as the smallest complex containing 
all simplexes in $\bigcup_{\sigma \in K} S \sigma$. By induction we define a sequence of complexes

$$
K_{1} \equiv K, \quad K_{n+1} \equiv S K_{n} .
$$

Later on we will also use the other special case of standard subdivision, called by us the regular standard subdivision (or shortly "regular subdivision"). In this case for a $q$-simplex $\sigma=\left(p_{0}, p_{1}, \ldots, p_{q}\right)$ we define the vertices of $S \sigma$ as in (1.33), but declare that the simplexes of $S \sigma$ are all similar to $\sigma$, i.e. each simplex in $S \sigma$ can be obtained by contraction of $\sigma$ by a factor $\frac{1}{2}$ and possibly a translation accompanied by rotation. Then we define a sequence of regular subdivisions by induction as in (1.35).

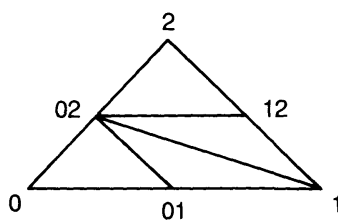

Whitney standard subdivision

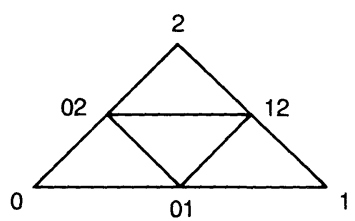

Regular standard subdivision

The standard subdivisions have the following important features:

a) Given a simplicial complex $K$, at most a finite number of shapes occur among the simplexes of $\bigcup_{n} K_{n}, K_{n} \equiv S_{n} K$.

b) The mesh $\eta$ of $S_{n} K$ has the property

$$
\eta\left(S_{n} K\right) \rightarrow 0 \text { as } n \rightarrow \infty,
$$

and there is a constant $0<c_{0}<\infty$ such that for any $n \in \mathbb{N}$,

$$
c_{0} \leqq \Theta\left(S_{n} K\right)
$$

$\Theta$ being the fullness defined by (1.32).

Let $W_{n}$ respectively $R_{n}$ denote Whitney respectively De Rham map associated to the complex $K_{n}$ defined in (1.35). Now we can formulate the basic results of simplicial approximation theory:

Theorem 1.3. Suppose $\left\{K_{n}\right\}_{n \in \mathbb{N}}$ is a family of smooth triangulations of a $C^{\infty}$ compact Riemannian manifold $M$, defined by standard subdivisions. Then we have

a) For any $\alpha \in \Lambda$

$$
W_{n} R_{n} \alpha \underset{n \rightarrow \infty}{\longrightarrow} \alpha \text { in } L^{2} \Lambda \text {. }
$$

b) Let $\left\{-\lambda_{l}^{c}, \phi_{l}^{c}\right\}$ be the eigenvalues and eigenvectors of a combinatorial Laplacian defined in $C\left(K_{n}\right)$, i.e.

$$
-\Delta^{c} \phi_{l}^{c}=\lambda_{l}^{c} \phi_{l}^{c}, \quad \lambda_{l}^{c} \geqq 0 .
$$

Then there is an indexing $\left\{-\lambda_{i}, \phi_{i}\right\}$ of eigenvalues and eigenvectors of a Laplacian 
$\Delta$ in continuum (with corresponding boundary conditions) so that

$$
\lambda_{l} \underset{n \rightarrow \infty}{\longrightarrow} \lambda_{l} \text {, }
$$

and

$$
W_{n} \phi_{l}^{c} \underset{n \rightarrow \infty}{\longrightarrow} \phi_{l} \text { in } L^{2} \Lambda .
$$

c) Let $G^{c}$ be the Green operator corresponding to the Laplacian $\Delta^{c}$ defined in $C\left(K_{n}\right)$. Then for any $\alpha \in \Lambda$,

$$
W_{n} G^{c} R_{n} \alpha \underset{n \rightarrow \infty}{\longrightarrow} G \alpha \quad \text { in } L^{2} \Lambda
$$

Proof.

a) This is proved in [Do] (see also [DoP] and, for functions $[\mathrm{StrF}]$ ).

b) This is proved in [DoP].

c) This follows essentially from a), b), cfr. also e.g. [Mü] (Th. 5.15).

\section{Remarks.}

1. In the above statements a), b) one can replace the particular approximation through the linear functions $W_{n} \phi_{l}^{c}$ by other approximations belonging to the quadratic form domain of $\Delta$.

2. In this paper we do not need the precise rate of convergence in a), b), c). Let us mention that this rate has been obtained for a), b) in [Do], [DoP] (see also (StrF]), and for c) in the work [M $\ddot{\text { in] }}$ (in the course of the proof of the Ray-Singer-conjecture on equivalence of analytic and combinatorial (Reidemeister-Franz) torsion. For the proof of the Ray-Singer-conjecture see also [Che]).

\section{The Simplicial Fields}

Let $K$ be a complex of a smooth triangulation of a $C^{\infty}$ compact connected Riemannian manifold $M$ and let $L \subset K$ be a subcomplex triangulating $\partial M$ (possibly empty). Let $0 \leqq q \leqq \operatorname{dim} M$.

Definition 2.1. A $q^{\text {th }}$-simplicial field is a map

given by

$$
\varphi: \mathbb{R}^{\operatorname{dim} C^{q}(K)} \rightarrow C^{q}(K)
$$

$$
\varphi:=\sum_{i} \varphi_{i} \sigma_{i},
$$

with the sum running over $i=1, \ldots, \operatorname{dim} C^{q}(K)$ and where $\sigma_{i}$ are $q$-simplexes of $K$, whereas $\varphi_{i}$ denotes the $i$-th coordinate function on $\mathbb{R}^{\operatorname{dim} C^{q}(K)}$.

For any $\sigma \in C^{q}(K)$ we set

$$
\varphi(\sigma):=\sum_{i} \varphi_{i}\left(\sigma_{i}, \sigma\right) .
$$

If the manifold $M$ has nonempty boundary $\partial M$, we can also define a $q$-simplicial field $\varphi_{D}$ with Dirichlet boundary conditions on $\partial M$. This field is by definition the map from $\mathbb{R}^{\operatorname{dim} C_{0}^{q}(K)}$ to $C_{0}^{q}(K)$ and is given by (2.3) with the summation restricted to $q$-simplexes $\sigma_{i} \notin L$. The field with Neumann boundary conditions on $\partial M$ is by definition identified with that given by Definition 2.1. 
By the very definition the following quadratic forms are well defined nonnegative real functions:

$$
\begin{aligned}
\left(d^{c} \varphi, d^{c} \varphi\right) & \equiv \sum_{i, j} \varphi_{i} \varphi_{j}\left(d^{c} \sigma_{i}, d^{c} \sigma_{j}\right), \\
(\varphi, \varphi) & \equiv \sum_{i, j} \varphi_{i} \varphi_{j}\left(\sigma_{i}, \sigma_{j}\right), \\
(\varphi, H \varphi) & \equiv \sum_{i, j} \varphi_{i} \varphi_{j}\left(\sigma_{i}, H \sigma_{j}\right) .
\end{aligned}
$$

For $\left(\lambda_{0}, m\right) \in\left\{\left(\mathbb{R}^{+} \times \mathbb{R}^{+}\right) \backslash(0,0)\right\}\left(\right.$ with $\left.\mathbb{R}^{+} \equiv[0, \infty)\right)$ we define

$$
\left(\varphi,\left[G^{c}\right]^{-1} \varphi\right) \equiv\left(d^{c} \varphi, d^{c} \varphi\right)+\lambda_{0}(\varphi, H \varphi)+m^{2}(\varphi, \varphi)
$$

and with $m \in \mathbb{R}^{+}$

$$
\left(\varphi_{D},\left[G_{D}^{c}\right]^{-1} \varphi_{D}\right) \equiv\left(d^{c} \varphi_{D}, d^{c} \varphi_{D}\right)+m^{2}\left(\varphi_{D}, \varphi_{D}\right) .
$$

Definition 2.2. A free $q$-simplicial measure is defined by

$$
\mu_{q}^{c}(\cdot) \equiv \frac{1}{Z^{c}} \int \prod_{i} d \varphi_{i}\left(e^{-(1 / 2)\left(\varphi\left[G^{c}\right]^{-1} \varphi\right)}\right)
$$

with $\prod_{i} d \varphi_{i}$ the Lebesgue measure on $\mathbb{R}^{\operatorname{dim} C^{q}(\boldsymbol{K})}$ and $Z^{c}$ a normalization factor making $\mu_{q}^{c}$ into a probability measure.

If $\partial M \neq \varnothing$, we define a free $q$-simplicial measure with Dirichlet boundary conditions on $\partial M$ by

$$
\mu_{q, D}^{c}(\cdot) \equiv \frac{1}{Z_{D}^{c}} \int \prod_{i} d \varphi_{D, i}\left(e^{-(1 / 2)\left(\varphi_{D}\left[G_{D}^{c}\right]^{-1} \varphi_{D}\right)}\right)
$$

with $\prod_{i} d \varphi_{D, i}$ the Lebesgue measure on $\mathbb{R}^{\operatorname{dim} C_{0}^{q}(K)}$ and $Z_{D}^{c}$ a corresponding normalization factor. If $\partial M \neq \varnothing$, then a $q$-simplicial free measure $\mu_{q, N}^{c}$ with Neumann boundary condition is by definition equal to (2.7). Since the quadratic forms (2.5) and (2.6) are strictly positive definite in the corresponding range of parameters, so the $q$-simplicial free measures in the above sense are well defined. If in a given context it is not necessary to mention the boundary conditions, we will use $\mu_{q}^{c}$ to denote any of the above defined probability measures.

Before we make a more detailed analysis of $q$-simplicial fields, let us note that they all converge to the corresponding $q$-fields in the continuum limit. To see this, let us consider a triangulation $K_{n}$ of $M$ given as the $n$-th standard subdivision of a smooth triangulation $K$ and define the following characteristic functionals:

$$
\Lambda^{q} \ni \alpha \mapsto I_{n}^{q}(\alpha):=\mu_{q}^{c}\left(e^{i \varphi\left(R_{n} \alpha\right)}\right)
$$

with $\mu_{q}^{c}$ the free $q$-simplicial measure defined above for the complex $K_{n}$. Using the properties of Whitney and de Rham maps one can see that the functional (2.9) is also well defined on the set

which is dense in $L^{2} \Lambda^{q}$.

$$
O^{(q)} \equiv \bigcup_{n^{\prime}} W C^{q}\left(K_{n^{\prime}}\right)
$$


Theorem 2.3. Let $\left\{K_{n}\right\}_{n \in \mathbf{N}}$ be a family of smooth triangulations of a compact $C^{\infty}$ Riemannian manifold $M$, defined by standard subdivisions. Then, for any $\alpha \in \Lambda^{q} \cup O^{(q)}$, $0 \leqq q \leqq \operatorname{dim} M$, we have

$$
\lim _{n \rightarrow \infty} I_{n}^{(q)}(\alpha)=\mu_{q}\left(e^{i \varphi(\alpha)}\right)
$$

where $\mu_{q}$ is the Gaussian measure on $\left(\Lambda^{q}\right)^{\prime}$ with mean zero and covariance

$$
\mu_{q}(\varphi(\alpha) \varphi(\tilde{\alpha})) \equiv\left(\alpha,\left(-\Delta+\lambda_{0} H+m^{2} I\right)^{-1} \tilde{\alpha}\right)_{L^{2} \Lambda^{q}}
$$

where $\alpha, \tilde{\alpha} \in \Lambda^{q}$.

Proof. The proof easily follows from Theorem $1.3(\mathrm{a}, \mathrm{b})$.

Suppose $\alpha, \tilde{\alpha} \in O^{(q)}$ i.e. there is some $n_{0} \in \mathbb{N}$ such that $\alpha, \tilde{\alpha} \in W_{n_{0}} C^{q}\left(K_{n_{0}}\right)$. Let $\mu_{q}^{c}$ be a free $q$-simplicial measure defined for a triangulation $K_{n}, K_{n_{0}} \subset K_{n}$. Let $\left\{-\lambda_{l}^{c}, \phi_{l}^{c}\right\}$ be eigenvalues and eigenvectors of a combinatorial Laplacian $\Delta^{c}$ on $C^{q}\left(K_{n}\right)$ ordered so that $\lambda_{l}^{c} \leqq \lambda_{l+1}^{c}$. It is assumed that the $\phi_{i}^{c}$ are normalized such that $\left(\phi_{l}^{c}, \phi_{l}^{c}\right)=1$. Then we have

$$
\begin{aligned}
\mu_{q}^{c}\left(\varphi\left(R_{n} \alpha\right) \varphi\left(R_{n} \tilde{\alpha}\right)\right)= & \sum_{\phi_{0}^{c} \in H^{(q)}}\left(R_{n} \alpha, \phi_{0}^{c}\right)\left(\lambda_{0}+m^{2}\right)^{-1}\left(\phi_{0}^{c}, R_{n} \tilde{\alpha}\right) \\
& +\sum_{l=1}^{\operatorname{dim} C^{(n)}\left(K_{n}\right)}\left(R_{n} \alpha, \phi_{l}^{c}\right)\left(\lambda_{l}^{c}+m^{2}\right)^{-1}\left(\phi_{l}^{c}, R_{n} \tilde{\alpha}\right) .
\end{aligned}
$$

We remark that $\lambda_{0}$ in the first term on the right-hand side of (2.13) is not an eigenvalue of $-\Delta^{c}$, but rather the parameter standing before the harmonic projector $H$ in Definition 2.2 of the measure $\mu_{q}^{c}$. If the space $H^{(q)}$ is empty then we can take $\lambda_{0}, m=0$ and by convention the first sum on the right-hand side of (2.13) is equal to zero. Take $N \in \mathbb{N}, \varepsilon>0$. Then using the fact that for $\alpha \in W_{n_{0}} C^{q}\left(K_{n_{0}}\right)$ we have

$$
W_{n} R_{n} \alpha=\alpha
$$

for all $n \geqq n_{0}$, and using Theorem $1.3(\mathrm{a}, \mathrm{b})$ we get the estimates

$$
\begin{gathered}
\left|\sum_{\phi_{0}^{c} \in H^{(q)}}\left(\alpha, W_{n} \phi_{0}^{c}\right)\left(\lambda_{0}+m^{2}\right)^{-1}\left(W_{n} \phi_{0}^{c}, \tilde{\alpha}\right)-\sum_{\phi_{0} \in H(q)}\left(\alpha, \phi_{0}\right)\left(\lambda_{0}+m^{2}\right)^{-1}\left(\phi_{0}, \tilde{\alpha}\right)\right|<\frac{\varepsilon}{4}, \\
\left|\sum_{l=1}^{N}\left(\alpha, W_{n} \phi_{l}^{c}\right)\left(\lambda_{l}^{c}+m^{2}\right)^{-1}\left(W_{n} \phi_{l}^{c}, \tilde{\alpha}\right)-\sum_{l=1}^{N}\left(\alpha, \phi_{l}\right)\left(\lambda_{l}+m^{2}\right)^{-1}\left(\phi_{l}, \tilde{\alpha}\right)\right|<\frac{\varepsilon}{4},
\end{gathered}
$$

provided $n \in \mathbb{N}$ is taken sufficiently big. We have also

$$
\left|\sum_{l=N+1}^{\operatorname{dim} C^{q}\left(K_{n}\right)}\left(R_{n} \alpha, \phi_{l}^{c}\right)\left(\lambda_{l}^{c}+m^{2}\right)^{-1}\left(\phi_{l}^{c}, R_{n} \tilde{\alpha}\right)\right| \leqq\left(\lambda_{N+1}^{c}\right)^{-1}\|\alpha\| \cdot\|\tilde{\alpha}\| \leqq 2 \cdot \lambda_{N+1}^{-1}\|\alpha\| \cdot\|\tilde{\alpha}\|
$$

if $n \in \mathbb{N}$ is sufficiently big, and

$$
\left|\sum_{l=N+1}^{\infty}\left(\alpha, \phi_{l}\right)\left(\lambda_{l}+m^{2}\right)^{-1}\left(\phi_{l}, \tilde{\alpha}\right)\right| \leqq \lambda_{N+1}^{-1}\|\alpha\| \cdot\|\tilde{\alpha}\| .
$$


Using the fact that each eigenvalue has finite multiplicity and the sequence of different eigenvalues of the Laplacian $-\Delta$ in the continuum diverges to infinity, we can estimate the sum of (2.17) and (2.18) by $\varepsilon / 2$, provided $N \in \mathbb{N}$ is taken sufficiently big, depending only on $\varepsilon$. Combining the above estimations we get that for any $\alpha, \tilde{\alpha} \in W_{n_{0}} C^{q}\left(K_{n_{0}}\right), n_{0} \in \mathbb{N}$,

$$
\left|\mu_{q}^{c}\left(\varphi\left(R_{n} \alpha\right) \varphi\left(R_{n} \tilde{\alpha}\right)\right)-\mu_{q}(\varphi(\alpha) \varphi(\tilde{\alpha}))\right|<\varepsilon
$$

if $n \in \mathbb{N}$ is taken sufficiently big.

This implies (2.11) for $\alpha \in O^{(q)}$. The proof for $\alpha \in \Lambda^{q}$ goes through the same arguments plus the use of Theorem 1.3a) about the convergence in $L^{2} \Lambda^{q}$ of $W_{n} R_{n} \alpha$ to $\alpha$.

\section{Inequalities for Scalar Simplicial Free Fields on a Manifold}

Let $M$ be a compact connected $C^{\infty}$ Riemannian manifold with boundary $\partial M$ (possibly empty). Let $K$ be a finite smooth triangulation of $M$ and let $L \subset K$ be a subcomplex triangulating $\partial M$. In this section we will investigate 0 -simplicial fields. In the case where $\partial M=\varnothing$ or where we take Neumann-boundary conditions the space $H^{(0)}$ of harmonic 0 -cochains is nonempty. Then we have the following explicit expression for the harmonic projection on $C^{0}(K)$ :

$$
H \varphi=\left(\frac{1}{|M|} \sum_{i} \varphi_{i}\left(\sigma_{i}, \mathbb{1}\right)\right) \sum_{j} \sigma_{j},
$$

where $|M|$ means Riemannian volume of $M$ and

$$
\mathbb{1} \equiv \sum_{j} \sigma_{j}
$$

with summation going over all $j$ such that $\sigma_{j} \in C^{0}(K)$. From (3.1) we get

$$
(\varphi, H \varphi)=\frac{1}{|M|}\left(\sum_{i} \varphi_{i}\left(\sigma_{i}, \mathbb{1}\right)\right)^{2} .
$$

This implies that the quadratic form $(\varphi, H \varphi)$ is a convex function of $\left\{\varphi_{i}\right\}$.

Note that by our definition the quadratic form $(\varphi, \varphi)$ is also a convex function of $\left\{\varphi_{i}\right\}$. For our further purposes let us introduce the following partial order in the space of probability measures on a finite dimensional vector space.

Definition 3.1. Let $\mu$ and $\tilde{\mu}$ be the probability measures on a topological vector space $V^{\prime}$. We say that $\tilde{\mu}$ is less than $\mu$ in the sense of Brascamp-Lieb if for any $f \in V$ and $n \in \mathbb{N}$

$$
\tilde{\mu}\left(|\varphi(f)|^{n}\right) \leqq \mu\left(|\varphi(f)|^{n}\right)
$$

with $\varphi(f): V^{\prime} \rightarrow \mathbb{R}: V^{\prime} \ni l \mapsto \varphi(f)(l):=l(f), V$ being dual to $V^{\prime}$. If (3.4) is satisfied we write

$$
\tilde{\mu} \underset{B-L}{\leqq} \mu .
$$

With the above notation we have 
Lemma 3.2 [Brascamp-Lieb]. Let $\tilde{\mu}$ be a perturbation of a Gaussian measure on a finite dimensional vector space by a log concave function, i.e.

$$
\tilde{\mu}(\cdot)=\frac{\mu\left(e^{-U} \cdot\right)}{\mu\left(e^{-U}\right)}
$$

for some convex function $U$. Then

$$
\tilde{\mu} \underset{B-L}{\leqq} \mu
$$

Using this Lemma and (3.3) we get easily

Proposition 3.3. Let $\mu_{0}^{c}$ be defined by (2.7) with $\lambda_{0} \geqq 0$ and $m_{0}>0$. Then

$$
\mu_{0}^{c} \leqq\left.\mu_{0}^{c}\right|_{\lambda_{0}=0}
$$

If $\lambda_{0}>0$, then $\left.\mu_{0}^{c}\right|_{m_{0}=0}$ is well defined and

$$
\mu_{0}^{c} \leqq\left.\mu_{0}^{c}\right|_{m_{0}=0}
$$

Let $\sigma \in K$ be a $D$-chain, $D \equiv \operatorname{dim} M$. Let $\partial \sigma \equiv \overline{S t \sigma} \backslash \sigma$ and denote by $\tilde{\chi}_{\partial \sigma}$ the characteristic function of the image of $(K \backslash \partial \sigma)$. Using these notations we define the following quadratic forms with $m_{0}^{2}>0$ :

$$
\begin{aligned}
\left(\varphi,\left[G_{N(\hat{\sigma} \sigma)}^{c}\right]^{-1} \varphi\right) \equiv & \sum_{i, j} \varphi_{i} \varphi_{j}\left(\tilde{\chi}_{\hat{c} \sigma} W d^{c} \sigma_{i}, \tilde{\chi}_{\hat{\sigma} \sigma} W d^{c} \sigma_{j}\right) \\
& +m_{0}^{2} \sum_{i, j} \varphi_{i} \varphi_{j}\left(\tilde{\chi}_{\partial \sigma} W \sigma_{i}, \tilde{\chi}_{\hat{\partial} \sigma} W \sigma_{j}\right)
\end{aligned}
$$

Thus the form $\left[G_{N(\hat{c} \sigma)}^{c}\right]^{-1}$ is obtained from the form $\left[G^{c}\right]^{-1}$ with $\lambda_{0} \equiv 0$, by removing a quadratic form supported in $\partial \sigma$. Equivalently we can get (3.10) by setting

$$
W \varphi^{N(\hat{\imath} \sigma)} \equiv\left(1-\tilde{\chi}_{\hat{c} \sigma}\right) W \varphi
$$

into $\left[G^{c}\right]^{-1}$.

Let $\mu_{N(\hat{c} \sigma)}^{c}$ denote the Gaussian probability measure (with Neumann boundary condition on $\partial \sigma$ ) having covariance given by the form $\left[G_{N(\partial \sigma)}^{c}\right]^{-1}$ in (3.10). Then again invoking Lemma 3.2 we get

Proposition 3.4. Let $\mu_{0}^{c}$ respectively $\mu_{N(\partial \sigma)}^{c}$ be a Gaussian measure corresponding to $\left[G^{c}\right]^{-1}$ respectively $\left[G_{N(\hat{\kappa} \sigma)}^{c}\right]^{-1}$ defined with $\lambda_{0}=0$ and $m_{0}>0$. Then

$$
\mu_{0}^{c} \underset{B-L}{\leqq} \mu_{N(\partial \sigma)}^{c}
$$

One can define also a form $\left[G_{N(\partial \sigma)}^{c}\right]^{-1}$ with Dirichlet boundary condition on $\partial \sigma$ by removing from $\left[G^{c}\right]^{-1}$ the terms of the form $\varphi_{i} \varphi_{j}\left[\left(d^{c} \sigma_{i}, d^{c} \sigma_{j}\right)+m^{2}\left(\sigma_{i}, \sigma_{j}\right)\right]$ with $\sigma_{i}, \sigma_{j} \in C^{0}(K)$ such that $\sigma_{i} \in \bar{\sigma}$ and $\sigma_{j} \in S t \sigma \backslash \bar{\sigma}$. Let $\mu_{D(\partial \sigma)}^{c}$ denote the corresponding measure. In the usual euclidean field theory on the lattice $\mathbb{Z}^{d}$ (see e.g. [GRS], [Si], [GJ]) one can relate the measure defined with Dirichlet boundary condition to a measure with free or Neumann boundary conditions by inequalities of the form (3.4). This is false in general for triangulations of Riemannian manifolds. Before 

discussing in more detail the obstructions, let us consider the following
example.

Example 3.5. Let $M \subset \mathbb{R}^{2}$ be a square of side $L \in \mathbb{N}$. We choose a triangulation of $M$ defined by the bonds in $\eta \mathbb{Z}^{2}$ and lines diagonals of unit squares in the direction $((0,0), \eta(-1,1))$.

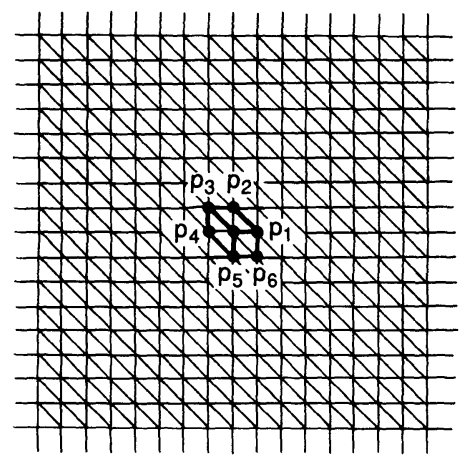

Let $\left\{g^{\mu \nu}\right\}$ be a (nondegenerate) metric in $M$ and let $g \equiv\left(\operatorname{det} g_{\mu \nu}\right)^{1 / 2}$, where det means determinant.

Let us compute matrix elements of the form (2.4). For that it is sufficient to compute corresponding scalar products in the simplex $\left(p_{0}, p_{1}, p_{2}\right)$, where $p_{0} \equiv(0,0)$, $p_{1} \equiv(\eta, 0), p_{2} \equiv(0, \eta)$ and use symmetry arguments.

In the 2-simplex $\sigma \equiv \sigma^{(2)} \equiv\left(p_{0}, p_{1}, p_{2}\right)$ we have the following barycentric coordinates

$$
\begin{aligned}
& W \sigma_{1}=\frac{x^{1}}{\eta} \\
& W \sigma_{2}=\frac{x^{2}}{\eta}, \\
& W \sigma_{0}=1-\frac{x^{1}}{\eta}-\frac{x^{2}}{\eta}
\end{aligned}
$$

where $\sigma_{i} \equiv \sigma_{i}^{(0)} \equiv\left(p_{i}\right)$ are 0 -simplexes.

Let us note that we have

$$
\begin{aligned}
& * d x^{1}=-g^{21} g d x^{1}+g^{11} g d x^{2}, \\
& * d x^{2}=-g^{22} g d x^{1}+g^{12} g d x^{2} .
\end{aligned}
$$

The volume form is then equal

$$
w \equiv g d x^{1} \wedge d x^{2} .
$$

Using this information we compute the part of matrix elements $\left(d W \sigma_{i}, d W \sigma_{j}\right)$ coming from the 2-simplex $\sigma$. With $\chi_{\sigma}$ being the characteristic function of the 
simplex $\sigma$ we have

$\left(\chi_{\sigma} d W \sigma_{1}, \chi_{\sigma} d W \sigma_{2}\right)=\int_{\sigma} \frac{1}{\eta} d x^{1} \wedge * \frac{1}{\eta} d x^{2}=\frac{1}{\eta^{2}} \int_{\sigma} g^{12} g d x^{1} \wedge d x^{2}$

$\left(\chi_{\sigma} d W \sigma_{0}, \chi_{\sigma} d W \sigma_{1}\right)=\int_{\sigma}\left(-\frac{1}{\eta} d x^{1}-\frac{1}{\eta} d x^{2}\right) \wedge * \frac{1}{\eta} d x^{1}=-\frac{1}{\eta^{2}} \int_{\sigma}\left(g^{11}+g^{21}\right) g d x^{1} \wedge d x^{2}$,

$\left(\chi_{\sigma} d W \sigma_{0}, \chi_{\sigma} d W \sigma_{2}\right)=\int_{\sigma}\left(-\frac{1}{\eta} d x^{1}-\frac{1}{\eta} d x^{2}\right) \wedge * \frac{1}{\eta} d x^{2}=-\frac{1}{\eta^{2}} \int_{\sigma}\left(g^{12}+g^{22}\right) g d x^{1} \wedge d x^{2}$

and

$$
\begin{aligned}
\left(\chi_{\sigma} d W \sigma_{0}, \chi_{\sigma} d W \sigma_{0}\right) & =\int_{\sigma}\left(-\frac{1}{\eta} d x^{1}-\frac{1}{\eta} d x^{2}\right) \wedge *\left(-\frac{1}{\eta} d x^{1}-\frac{1}{\eta} d x^{2}\right) \\
& =\frac{1}{\eta^{2}} \int_{\sigma}\left(g^{11}+g^{12}+g^{21}+g^{22}\right) g d x^{1} \wedge d x^{2} \\
\left(\chi_{\sigma} d W \sigma_{1}, \chi_{\sigma} d W \sigma_{1}\right) & =\int_{\sigma} \frac{1}{\eta} d x^{1} \wedge * \frac{1}{\eta} d x^{1} \\
& =\frac{1}{\eta^{2}} \int_{\sigma} g^{11} g d x^{1} \wedge d x^{2} \\
\left(\chi_{\sigma} d W \sigma_{2}, \chi_{\sigma} d W \sigma_{2}\right) & =\int_{\sigma} \frac{1}{\eta} d x^{2} \wedge * \frac{1}{\eta} d x^{2} \\
& =\frac{1}{\eta^{2}} \int_{\sigma} g^{22} g d x^{1} \wedge d x^{2} .
\end{aligned}
$$

Using (3.19)-(3.24) one can easily determine also the other scalar products $\left(\chi_{\sigma^{\prime}} d W \sigma_{i}, \chi_{\sigma^{\prime}} d W \sigma_{j}\right)$, for $\sigma^{\prime}$ being 2-simplexes in $S t \sigma_{0}$ and $\sigma_{i}, \sigma_{j} \in \overline{S t \sigma_{0}}$. Combining these results and numbering 2-simplexes in $\overline{S t \sigma_{0}}$ anticlockwise beginning from $\sigma_{1}^{(2)} \equiv \sigma^{(2)} \equiv\left(p_{0}, p_{1}, p_{2}\right)$, we get:

$$
\begin{aligned}
\left(d W \sigma_{0}, d W \sigma_{0}\right)= & \frac{1}{\eta^{2}} \int_{\left(\sigma_{1}^{(2)}+\sigma_{4}^{(2)}\right)}\left(g^{11}+2 g^{12}+g^{22}\right) g d x^{1} \wedge d x^{2} \\
& +\frac{1}{\eta^{2}} \int_{\left(\sigma_{3}^{(2)}+\sigma_{5}^{(2)}\right)} g^{11} g d x^{1} \wedge d x^{2}+\frac{1}{\eta^{2}} \int_{\left(\sigma_{2}^{(2)}+\sigma_{6}^{(2)}\right)} g^{22} g d x^{1} \wedge d x^{2},
\end{aligned}
$$

and

$$
\begin{aligned}
& \left(d W \sigma_{0}, d W \sigma_{1}\right)=-\frac{1}{\eta^{2}} \int_{\left(\sigma_{1}^{(2)}+\sigma_{6}^{(2)}\right)}\left(g^{11}+g^{21}\right) g d x^{1} \wedge d x^{2} \\
& \left(d W \sigma_{0}, d W \sigma_{2}\right)=-\frac{1}{\eta^{2}} \int_{\left(\sigma_{1}^{(2)}+\sigma_{2}^{(2)}\right)}\left(g^{12}+g^{22}\right) g d x^{1} \wedge d x^{2}, \\
& \left(d W \sigma_{0}, d W \sigma_{3}\right)=\frac{1}{\eta^{2}} \int_{\left(\sigma_{2}^{(2)}+\sigma_{3}^{(2)}\right)} g^{12} g d x^{1} \wedge d x^{2} .
\end{aligned}
$$


The remaining matrix elements $\left(d W \sigma_{0}, d W \sigma_{j}\right)$ are determined similarly.

Now consider matrix elements $\left(\sigma_{i}, \sigma_{j}\right)$ of the form $(\varphi, \varphi)$ given in $(2.4 \mathrm{~b})$. One can easily see that

$$
\left(\sigma_{i}, \sigma_{j}\right)=0 \text { if } \sigma_{i} \notin \overline{\operatorname{St} \sigma_{j}}
$$

and

$$
\left(\sigma_{i}, \sigma_{j}\right)>0 \text { if } \sigma_{i} \in \overline{S t \sigma_{j}} .
$$

In particular we have here strictly positive off diagonal elements.

Let us note that the form $(\varphi, H \varphi)$ given by (3.3) has all off diagonal elements strictly positive and contrary to the forms $(\varphi, \varphi)$ or $\left(d^{c} \varphi, d^{c} \varphi\right)$ is nonlocal.

Now let us come back to the general case. We can use the form

$$
\left(\varphi_{D},\left[G_{D}^{c}\right]^{-1} \varphi_{D}\right)=\left(d^{c} \varphi_{D}, d^{c} \varphi_{D}\right)
$$

to define a corresponding combinatorial free measure $\mu_{D}^{c}$ with Dirichlet boundary conditions on $\partial M$ (and mass $m_{0} \equiv 0$ ) (in the sense that $\mu_{D}^{c}$ is determined as the Gaussian measure with mean zero and covariance $G_{D}^{c}$ ). By analysing formulas (3.25-3.28) of Example 3.5 we can draw the following conclusions, not only true for the example but rather true in any dimension:

Proposition 3.6. Let $M$ be a cube in $\mathbb{R}^{d}$ with edges of length l. Let $K$ be its regular triangulation defined by a lattice $\delta \mathbb{Z}^{d},(l / \delta \in \mathbb{N})$, and parallel hyperplanes dividing each cube of the lattice into two d-simplexes. The measure $\mu_{D}^{c}$ given by the form (3.30) is identical with the free measures $\mu_{\delta}^{D}$ with Dirichlet boundary conditions on $\partial M$ of euclidean scalar massless field theory on the lattice $\delta \mathbb{Z}^{d}$ iff

$$
g^{\mu v}=\delta^{\mu \nu} .
$$

Let us remind (see e.g. [GIJ], [Si]) that a probability measure $\mu$ on $\mathbb{R}^{D}$ satisfies GKS inequalities if for any $n, m \in \mathbb{N}, i_{j}, i_{j^{\prime}}=1, \ldots, D$,

$$
\begin{gathered}
\mu\left(\prod_{j=1}^{n} \varphi_{i_{j}}\right) \geqq 0, \\
\mu\left(\prod_{j=1}^{n} \varphi_{i_{j}}, \prod_{j^{\prime}=1}^{m} \varphi_{i_{j^{\prime}}}\right) \geqq 0,
\end{gathered}
$$

where $\mu(f, g) \equiv \mu(f g)-\mu(f) \mu(g)$. One says that $\mu$ satisfies $\mathrm{FKG}$ inequalities if for any nondecreasing functions $F$ and $G$

$$
\mu(F, G) \geqq 0 .
$$

The inequalities (3.31) and (3.32) are called ferromagnetic inequalities. It is known (see e.g. [GlJ], [Si]) that for a Gaussian measure $\mu_{0}$ with mean zero and covariance matrix $C_{i j}(i, j=1, \ldots, D)$ to satisfy $(3.31)$ is necessary and sufficient that $C_{i j} \geqq 0$ (cfr. [Le]). To satisfy (3.31) it is sufficient to have the strong ferromagneticity condition

$$
\left(C^{-1}\right)_{i j} \leqq 0 \quad \text { for } \quad i \neq j
$$


(see e.g. [Gi], [FKG], [Si], [GlJ]). We also observe that the GKS inequalities $(3.31 \mathrm{a}, \mathrm{b})$ hold in all cases, for the general free measure $\mu_{0}$ as defined in Sect. 2. From Example 3.5 we see that to have ferromagnetic inequalities it is sufficient to require

$$
\begin{aligned}
g^{12} & \leqq 0, \\
g^{i j}+g^{i i} & \geqq 0 \text { for } i \neq j .
\end{aligned}
$$

In particular for the metrices

$$
g^{11}=1, \quad g^{12}=g^{21}= \pm 2, \quad g^{22}=4+\varepsilon, \varepsilon>0
$$

the condition (3.33) does not hold.

We remark that given a constant metric (3.36) one can get (3.33) by "passing to corresponding normal coordinates," what in our case means a correct choice of triangulation. These considerations can be extended to the case $D>2$. Let us note that since off diagonal elements $\left(\sigma_{i}, \sigma_{j}\right), \sigma_{i} \in \overline{S t \sigma_{j}}, i \neq j$ of the quadratic form $(\varphi, \varphi)$ are strictly positive, inclusion of a mass term in the definition of free 0 -simplicial measure always destroys the strong ferromagneticity condition (3.33). The term $\lambda_{0}(\varphi H \varphi)$ has the same effect if one wants to consider a measure with boundary conditions other than Dirichlet. Motivated by Euclidean field theory on lattice one can try to cure this drawback by taking instead of the term $(\varphi, \varphi)$ the other local mass term defined by

$$
\varphi^{2}(K)=\sum_{i} \varphi_{i}^{2}\left(\sigma_{i}, \mathbb{1}\right) .
$$

In this case we have to investigate the continuum limit once again. We postpone that to the next section.

Now let $\tilde{\mu}_{0}^{c}$ be defined as in (2.7) or (2.8) but with $m^{2} \varphi^{2}(K)$ instead of $m^{2}(\varphi, \varphi)$ respectively $m^{2}\left(\varphi_{D}, \varphi_{D}\right)$. Then we have

\section{Proposition 3.7.}

$$
\tilde{\mu}_{0}^{c} \leqq \mu_{B}^{c}
$$

Proof. We have from (3.37)

$$
\begin{aligned}
\varphi^{2}(K)-(\varphi, \varphi) & =\sum_{i} \varphi_{i}^{2}\left(\sigma_{i}, \mathbb{1}\right)-\sum_{i, j} \varphi_{i} \varphi_{j}\left(\sigma_{i}, \sigma_{j}\right) \\
& =\sum_{i, j} \varphi_{i}^{2}\left(\sigma_{i}, \sigma_{j}\right)-\sum_{i, j} \varphi_{i} \varphi_{j}\left(\sigma_{i} \sigma_{j}\right) \\
& =\sum_{i} \varphi_{i}^{2}\left(\sigma_{i}, \sum_{j: j \neq i} \sigma_{j}\right)-\sum_{i \neq j} \varphi_{i} \varphi_{j}\left(\sigma_{i}, \sigma_{j}\right) .
\end{aligned}
$$

We can write this in the form

$$
\varphi^{2}(K)-(\varphi, \varphi)=\sum_{i \neq j}\left(\varphi_{i}-\varphi_{j}\right)^{2}\left(\sigma_{i}, \sigma_{j}\right) .
$$

Since $\left(\sigma_{i}, \sigma_{j}\right) \geqq 0,(3.40)$ is a convex function and use of Lemma 3.2 ends the proof. 
We would like to close the section by one more comparison lemma, we will use later on. Let $K$ be a smooth triangulation of a $C^{\infty}$ manifold $M$ of dimension $D$. Let $\left\{K_{n}\right\}$ be the family of triangulations constructed by standard subdivisions of a smooth triangulation $K$. Let $\sigma$ be a $D$ simplex in $K$. After applying if necessary a smooth affine diffeomorphism we can and do identify $\sigma$ with a standard $D$-simplex in $\mathbb{R}^{D}$. Then all $D$-simplexes $\sigma_{n} \in K_{n}, \sigma_{n} \subset \sigma$ are obtained by a scaling with a factor $2^{-n}$ and, if necessary, by translation and rotation. In this case the 0 -simplexes $\sigma_{n}^{(0)} \in K_{n} \cap \sigma$ are the vertexes of the lattice $2^{-n} \mathbb{Z}^{D}$. We note that by our assumption a metric $\left\{g^{\mu \nu}\right\}$ on $M$ is nondegenerate, so there is a number $1 \leqq \lambda<\infty$ such that in the sense of quadratic forms we have

$$
\lambda^{-1} \delta^{\mu \nu} \leqq g^{\mu \nu}(x) \leqq \lambda \delta^{\mu \nu}
$$

for any $x \in h(\sigma) \subset M, h$ being a triangulation homeomorphism. We now have the following

Proposition 3.8. Let $\tilde{\mu}_{N(\partial \sigma), r}^{c}$ denote a Gaussian measure defined on $\mathbb{R}^{\operatorname{dim} C^{0}\left(K_{n} \cap \bar{\sigma}\right)}$ with covariance given by the quadratic form

$$
\left[G_{r, \delta}^{c}\right]^{-1} \equiv r^{(1+(D / 2))}\left\{\left(d^{c} \varphi, d^{c} \varphi\right)_{\delta}+m^{2}(\varphi, \varphi)_{\delta}\right\},
$$

where $m^{2}, r^{2}>0$ and $\delta$ indicates the use of diagonal metric $\delta^{\mu v}$ in the definition of corresponding quadratic forms. Let $\mu_{N(\partial \sigma)}^{c}$ respectively $\mu_{0}^{c}$ be the restriction to $\mathbb{R}^{\operatorname{dim} C^{0}\left(K_{n} \cap \sigma\right)}$ of the free 0-simplicial measure with Neumann boundary conditions on $\partial \sigma$ respectively without boundary conditions, defined with $m>0, \lambda_{0} \equiv 0$ and metric $\left\{g^{\mu \nu}\right\}$ satisfying (3.41). Then

$$
\mu_{0}^{c} \underset{B-L}{\leqq} \mu_{N(\partial \sigma)}^{c} \leqq \tilde{\mu}_{N(\partial \sigma), i^{-1}}^{c}
$$

and if additionally $\lambda_{0}$ in the definition of $\mu_{N(\partial \sigma)}^{c}$ is equal to zero we have

$$
\tilde{\mu}_{N(\partial \sigma), i}^{c} \leqq \mu_{B(\partial \sigma)}^{c}
$$

Proof. If (3.41) is satisfied, then we have for any $D$-simplex $\sigma_{n} \in K_{n} \cap \sigma$,

$$
\begin{aligned}
\lambda^{(-1+(D / 2))}\left(\chi_{\sigma_{n}} W d^{c} \varphi, \chi_{\sigma_{n}} W d^{c} \varphi\right)_{\delta} & \leqq\left(\chi_{\sigma_{n}} W d^{c} \varphi, \chi_{\sigma_{n}} W d^{c} \varphi\right)_{g} \\
& \leqq \lambda^{(1+(D / 2))}\left(\chi_{\sigma_{n}} W d^{c} \varphi, \chi_{\sigma_{n}} W d^{c} \varphi\right)_{\delta}
\end{aligned}
$$

and

$$
\begin{aligned}
\lambda^{-(D / 2)}\left(\chi_{\sigma_{n}} W \varphi, \chi_{\sigma_{n}} W \varphi\right)_{\delta} & \leqq\left(\chi_{\sigma_{n}} W \varphi, \chi_{\sigma_{n}} W \varphi\right)_{g} \\
& \leqq \lambda^{D / 2}\left(\chi_{\sigma_{n}} W \varphi, \chi_{\sigma_{n}} W \varphi\right)_{\delta}
\end{aligned}
$$

This implies if $\lambda_{0}=0$ the inequalities

$$
\left[G_{\lambda^{-1}, \delta}^{c}\right]^{-1} \leqq\left[G_{g}^{c}\right]^{-1} \leqq\left[G_{\lambda, \delta}^{c}\right]^{-1}
$$

and use of Lemma 3.2 ends the proof for $\lambda_{0}=0$. Now additional use of inequality (3.8) in Proposition 3.3 proves the validity of (3.43).

Remark. The merit of Proposition 3.8 is that locally we can bound expectations of the free 0 -simplicial measure $\mu_{0}^{c}$ with arbitrary (nondegenerate) metric by 
expectations with a measure corresponding to usual free Euclidean field rescaled by some finite factor.

\section{Local Interactions for Simplicial Fields}

In this section we would like to discuss local functions of the $q$-simplicial free field. We take a compact smooth Riemannian manifold $M$ of dimension $D$ and its smooth finite triangulation $K$. A possible definition of local functions of the field, natural from the point of view of conventional Euclidean field theory on lattice and possible ferromagnetic correlation inequalities, is the following

Definition 4.1. For $N \in \mathbb{N}$ and $f^{c} \in C^{q}(K)$ a Wick $N^{\text {th }}$ monomial $: \varphi^{N}:\left(f^{c}\right)$ of the $q$-simplicial free field is defined by

$$
: \varphi^{N}:\left(f^{c}\right) \equiv \sum_{i}: \varphi_{i}^{N}:_{\mu_{q}^{c}}\left(\sigma_{i}, f^{c}\right)
$$

where $::_{\mu_{q}^{c}}^{c}$ means the normal ordering with respect to a free $q$-simplicial measure $\mu_{q}^{c}$ and the summation runs over all $q$-simplexes $\sigma_{i} \in K$.

For a 0 -simplicial field one can consider also the following alternative definition of Wick $N^{\text {th }}$-monomial. Let us set for a 0 -simplex $\sigma_{i} \in K$ :

$$
\tilde{\sigma}_{i} \equiv\left(\int * W \sigma_{i}\right)^{-1} \sigma_{i}
$$

This definition is such that the sequence $W \tilde{\sigma}_{i}$ converges to the delta function (in the sense of distributions) in the formal continuum limit.

Definition 4.2. For $N \in \mathbb{N}$ and $f^{c} \in C^{0}(K)$ we set

$$
\vdots \varphi^{N}:\left(f^{c}\right) \equiv \sum_{j}: \varphi\left(\tilde{\sigma}_{j}\right)^{N}{: \mu_{0}^{c}}^{c}\left(\sigma_{j}, f^{c}\right)
$$

where $\varphi\left(\tilde{\sigma}_{j}\right)$ is given by (2.3). We call $\vdots \varphi^{N}:$ the triple dots $N^{\text {th }}$ Wick power. Let us note that by the very definitions we have

$$
\mu_{q}^{c}\left(: \varphi^{N}:\left(f^{c}\right)\right)^{2}=\sum_{i, j}\left(f^{c}, \sigma_{i}\right)\left(\mu_{q}^{c} \varphi_{i} \varphi_{j}\right)^{N}\left(\sigma_{j}, f^{c}\right),
$$

and for the 0 -simplicial field

$$
\mu_{0}^{c}\left(\vdots \varphi^{N}:\left(f^{c}\right)\right)^{2}=\sum_{i, j}\left(f^{c}, \sigma_{i}\right)\left(\tilde{\sigma}_{i}, G^{c} \tilde{\sigma}_{j}\right)^{N}\left(\sigma_{j}, f^{c}\right) .
$$

Note also that for $N=1$ the right-hand side of (4.4) is different from $\mu_{0}^{c}\left(\varphi\left(f^{c}\right)\right)^{2}=$ $\left(f^{c}, G^{c} f^{c}\right)$, but as one can easily see in the continuum limit with $f^{c}=R f$ the limit of both formulae is the same. It is clear that the local functions given in Definition 4.1 and Definition 4.2 are in $L^{2 t}\left(\mu_{q}^{c}\right)$ with corresponding $0 \leqq q \leqq D$ and $1 \leqq t<\infty$. Moreover for $q=0$ we have

Proposition 4.3. Let $\left\{K_{n}\right\}_{n \in \mathbb{N}}$ be a family of smooth triangulations of a two dimensional compact Riemannian manifold $M$ defined by standard regular subdivisions of the given smooth triangulation $K$. Let $\mu_{0}^{c}$ be a free 0-simplicial measure defined in Sect. 2 , with $m_{0}>0$. Then for any $f \in \Lambda^{0}, N \in \mathbb{N}$ and $1 \leqq t<\infty$.

$$
\mu_{0}^{c}\left(: \varphi^{N}:\left(R_{n} f\right)\right)^{t} \leqq C_{N, t}
$$


with a constant $0<C_{N, t}<\infty$ independent of $\left\{K_{n}\right\}$. Moreover there is a constant $0<\alpha_{0}$ (independent of the metric) such that for any $0<\alpha \leqq \alpha_{0}$ the function

$$
: e^{\alpha \varphi}:\left(R_{n} f\right)=\sum_{N=0}^{\infty} \frac{\alpha^{n}}{n !}: \varphi^{N}:\left(R_{n} f\right)
$$

is well defined in $L^{2}\left(\mu_{0}^{c}\right)$ and we have

$$
\mu_{q}^{c}\left(: e^{\alpha \varphi}:\left(R_{n} f\right)\right)^{2}<C
$$

with a constant $0<C<\infty$ independent of $\left\{K_{n}\right\}$.

The same holds for the local functions given by Definition 4.2.

Proof. By hypercontractive estimates for Gaussian measures [Ne] (see also [Gr1], [Si]) to prove (4.5) it is sufficient to show it for $t=2$. It is also sufficient to consider a localised problem in a $D$-simplex $\sigma \in K$, i.e. to consider $\chi_{\sigma} f$ instead of $f \in \Lambda^{q}$ (where $\chi_{\sigma}$ is characteristic function of the $h(\sigma)$-image of the simplex $\sigma$ ). We shall also need the following lemma, which is a consequence of some considerations in [FröZe]:

Lemma 4.4. Let $\mu, \mu^{\prime}$ be Gaussian measures on $\mathbb{R}^{d}$ such that

$$
\mu \underset{B-L}{\leqq} \mu^{\prime}
$$

For $\alpha \in \mathbb{R}^{d}$ and $N \in \mathbb{N}$, let $: \varphi^{N}:{ }_{\mu}(a)$ be the local function given by

$$
: \varphi^{N}:_{\mu}(a) \equiv \sum_{i=1, \ldots, d}: \varphi_{i}^{N}:_{\mu} a_{i}
$$

with $:{ }_{\mu}$ normal ordering with respect to $\mu$. Then

$$
\mu\left(: \varphi^{N}:_{\mu}(a)\right)^{2} \leqq \mu^{\prime}\left(: \varphi^{N}:_{\mu^{\prime}}(a)\right)^{2} .
$$

Proof. From (4.8) we have in particular, that in the sense of quadratic forms

$$
\mu \varphi_{i} \varphi_{j} \leqq \mu^{\prime} \varphi_{i} \varphi_{j}
$$

Then from the definition (4.9) we have

$$
\begin{aligned}
\mu\left(: \varphi^{N}:{ }_{\mu}(a)\right)^{2} & =\sum_{i, j} a_{i}\left(\mu \varphi_{i} \varphi_{j}\right)^{N} a_{j} \\
& =\bigotimes_{l=1}^{N} \mu_{l} \sum_{i, j} a_{i} \prod_{l=1}^{N} \varphi_{i}^{(l)} \prod_{l=1}^{N} \varphi_{j}^{(l)} a_{j}
\end{aligned}
$$

with $\mu_{l} \equiv \mu$ for $l=1, \ldots, N$.

The right-hand side of (4.12) can be written as follows

$$
\mu\left(: \varphi^{N}:{ }_{\mu}(a)\right)^{2}=\bigotimes_{l=2}^{N} \mu_{l}\left[\sum_{i, j}\left(a_{i} \prod_{l=2}^{N} \varphi_{i}^{(l)}\right) \mu \varphi_{i}^{(1)} \varphi_{j}^{(1)}\left(a_{j} \prod_{l=2}^{N} \varphi_{j}^{(l)}\right)\right] .
$$

Now application of (4.11) to the square bracket on the right-hand side of (4.13) gives

$$
\mu\left(: \varphi^{N}:_{\mu}(a)\right)^{2} \leqq \mu^{\prime}\left(\bigotimes_{l=2}^{N} \mu_{l} \sum_{i, j}\left(a_{i} \varphi_{i}^{(1)}\right) \prod_{l=2}^{N} \varphi_{i}^{(l)} \prod_{l=2}^{N} \varphi_{j}^{(l)}\left(a_{j} \varphi_{j}^{(1)}\right)\right)
$$

with $\varphi_{i}^{(1)}$ denoting the integration variable. Now we can apply the same arguments 
to the round bracket on the right-hand side of (4.14), writing it in the form

$$
\bigotimes_{l=3}^{N} \mu_{l}\left[\sum_{i, j}\left(a_{i} \varphi_{i}^{(1)} \prod_{l=3}^{N} \varphi_{i}^{(l)}\right) \mu \varphi_{i}^{(2)} \varphi_{j}^{(2)}\left(a_{j} \varphi_{j}^{(1)} \prod_{i=3}^{N} \varphi_{j}^{(l)}\right)\right] .
$$

Iteration of this procedure leads to

$$
\begin{aligned}
\mu\left(: \varphi^{N}:_{\mu}(a)\right)^{2} & =\bigotimes_{l=1}^{N} \mu^{\prime}\left(\sum_{i, j} a_{i} \prod_{i=1}^{N} \varphi_{i}^{(l)} \prod_{i=1}^{N} \varphi_{j}^{(l)} a_{j}\right) \\
& =\sum_{i, j} a_{i}\left(\mu^{\prime} \varphi_{i} \varphi_{j}\right)^{N} a_{j} \\
& =\mu^{\prime}\left(: \varphi^{N}:_{\mu^{\prime}}(a)\right)^{2}
\end{aligned}
$$

which ends the proof of the lemma.

Now we come back to the proof of Proposition 4.3. From Proposition 3.3, Proposition 3.4 and Proposition 3.8 we have

$$
\mu_{0}^{c} \leqq \tilde{\mu}_{N(\partial \sigma), \lambda^{-1}}^{c}
$$

with $1 \leqq \lambda<\infty$ such that in the sense of quadratic forms, for any $x \in h(\sigma)$,

$$
\lambda^{-1} \delta^{\mu v} \leqq g^{\mu v}(x),
$$

and the measure $\mu_{0}^{c}$ is a 0 -simplicial free measure defined on $\mathbb{R}^{\operatorname{dim} C^{0}\left(K_{n}\right)}$ with metric $\left\{g^{\mu \nu}\right\}$, whereas $\tilde{\mu}_{N(\partial \sigma), \lambda^{-1}}^{c}$ is the euclidean measure defined in Proposition 3.8. Using (4.17) and Lemma 4.4 we get

$$
\begin{aligned}
\mu_{0}^{c}\left(: \varphi^{N}:_{\mu_{0}^{c}}\left(R_{n} \chi_{\sigma} f\right)\right)^{2} & \leqq \tilde{\mu}_{N(\partial \sigma), \lambda^{-1}}^{c}\left(: \varphi^{N}:_{\lambda}\left(R_{n} \chi_{\sigma} f\right)\right)^{2} \\
& =\lambda^{4 N} \tilde{\mu}_{N(\partial \sigma), 1}^{c}\left(: \varphi^{N}:_{1}\left(R_{n} \chi_{\sigma} f\right)\right)^{2}
\end{aligned}
$$

with normal ordering on the right-hand side of inequality (4.19) with respect to the measure $\tilde{\mu}_{N(\partial \sigma), \lambda^{-1}}^{c}$ and on the right-hand side of the latter equality with respect to $\tilde{\mu}_{N(\partial \sigma), 1}^{c}$.

Since the expectations on the right-hand side of (4.19) are known to converge (see e.g. [GRS], [Si], [GlJ]) as $n \rightarrow \infty$ and moreover there is a constant $0<C_{0}<\infty$ independent of $\left\{K_{n}\right\}$ such that

$$
\tilde{\mu}_{N(\partial \sigma), 1}^{c}\left(: \varphi^{N}:\left(R_{n} \chi_{\sigma} f\right)\right)^{2} \leqq N ! C_{0}^{N},
$$

so we get (4.5) and (4.7) (using additionally the orthogonality of Wick monomials of different degrees). This ends the proof of Proposition 4.3 for the functions $: \varphi^{N}$ : given by Definition 4.1 .

Let us see what one can do for the triple dots Wick powers given by Definition 4.2. First of all let us note that

Hence

$$
\left(\tilde{\sigma}_{i}, G^{c} \tilde{\sigma}_{j}\right)=\sum_{k, k^{\prime}}\left(\tilde{\sigma}_{i}, \sigma_{k}\right) \mu_{0}^{c} \varphi_{k} \varphi_{k^{\prime}}\left(\sigma_{k^{\prime}}, \tilde{\sigma}_{j}\right)
$$

$$
\begin{aligned}
\mu_{0}^{c}\left(\vdots \varphi^{N}:(R f)\right)^{2} & =\sum_{i, j}\left(R f, \sigma_{i}\right)\left(\tilde{\sigma}_{i} G^{c} \tilde{\sigma}_{j}\right)^{N}\left(\sigma_{j}, R f\right) \\
& =\sum_{i, j}\left(R f, \sigma_{i}\right) \sum_{k_{1} k_{1}^{\prime}, \ldots, k_{N} k_{N}^{\prime}} \prod_{i=1}^{N}\left[\left(\tilde{\sigma}_{i}, \sigma_{k_{i}}\right) \mu_{0}^{c} \varphi_{k_{i}} \varphi_{k_{i}^{\prime}}\left(\sigma_{k_{i}^{\prime}}, \tilde{\sigma}_{j}\right)\right]\left(\sigma_{j}, R f\right) .
\end{aligned}
$$


Now let us write the right-hand side of (4.22) in form similar (4.13) from the proof of Lemma 4.4. We have

with

$$
\mu_{0}^{c}\left(\vdots \varphi^{N}:(R f)\right)^{2}=\bigotimes_{i=2}^{N} \mu_{i}\left(\sum_{k_{1} k_{1}^{\prime}} F_{k_{1}}^{(1)} \mu_{0}^{c} \varphi_{k_{1}}^{(1)} \varphi_{k_{1}^{\prime}}^{(1)} F_{k_{1}^{\prime}}^{(1)}\right)
$$

$$
F_{k_{1}}^{(1)} \equiv \sum_{i, k_{2}, \ldots, k_{N}}\left(R f, \sigma_{i}\right) \prod_{l=2}^{N}\left[\left(\tilde{\sigma}_{i}, \sigma_{k_{l}}\right) \varphi_{k_{l}}^{(l)}\right]
$$

By the same induction arguments as in the proof of Lemma 4.4, if "on $\sigma$ " (in the sense of (4.17))

$$
\mu_{0}^{c} \leqq \tilde{\mu}_{N(\partial \sigma), \lambda^{-1}}^{c}
$$

then for supp $f \subset \sigma$

$$
\mu_{0}^{c}\left(\vdots \varphi^{N}:(R f)\right)^{2} \leqq \sum_{i, j}\left(R f, \sigma_{i}\right)\left(\sum_{k, k^{\prime}}\left(\tilde{\sigma}_{i}, \sigma_{k}\right) \tilde{\mu}_{N(\partial \sigma), \lambda^{-1}}^{c}\left(\varphi_{k} \varphi_{k^{\prime}}\right)\left(\sigma_{k^{\prime}}, \tilde{\sigma}_{j}\right)\right)^{N} \cdot\left(\sigma_{j}, R f\right) .
$$

This ends the proof of our Proposition 4.3 for the case of local functions given by Definition 4.2 .

Remark. It should be possible to adapt a recent result proven in the continuum by S. Kusuoka $[\mathrm{Ku}]$ to prove that: $e^{\alpha \varphi}:\left(R_{n} f\right) \in L^{1+\varepsilon}\left(\mu_{0}^{c}\right)$ for $\alpha<\alpha_{1}$ and some $\alpha_{1}>\alpha_{0}, \varepsilon>0\left(\alpha_{0}\right.$ as in (4.6)), uniformly in the mesh $\eta$ and similarly for $e^{\alpha \varphi}:\left(R_{n} f\right)$.

\section{Continuum Limit}

Let $\left\{K_{n}\right\}_{n \in \mathbb{N}}$ be a family of smooth triangulations of $M$, constructed by standard regular subdivisions of $K$. In Sect. 2 we saw that for any $0 \leqq q \leqq D$,

$$
\lim _{n \rightarrow \infty} \mu_{q}^{c}\left(e^{i \varphi\left(R_{n} \alpha\right)}\right)=\mu_{q}\left(e^{i \varphi(\alpha)}\right)
$$

For the considered measures, this is equivalent to

$$
\lim _{n \rightarrow \infty} \mu_{q}^{c}\left(\prod_{i=1}^{N} \varphi\left(R_{n} \alpha_{i}\right)\right)=\mu_{q}\left(\prod_{i=1}^{N} \varphi\left(\alpha_{i}\right)\right)
$$

for any $N \in \mathbb{N}$ and $\alpha_{i} \in \Lambda^{q}, i=1, \ldots, N$. In the present section we would like to consider the continuum limit in the case when $D=2$ and $q=0$, for expectations containing Wick powers. Hence from now on we will take $D=2$ and $q=0$.

We have

Proposition 5.1. For any $N, N^{\prime} \in \mathbb{N}$ and $f, f_{i} \in C^{\infty}(M), i=1, \ldots, N^{\prime}$,

$$
\lim _{n \rightarrow \infty} \mu_{0}^{\mathrm{c}}\left(: \varphi^{N}:\left(R_{n} f\right): \prod_{i=1}^{N^{\prime}} \varphi\left(R_{n} f_{i}\right):\right)=\mu_{0}\left(: \varphi^{N}:(f): \prod_{i=1}^{N^{\prime}} \varphi\left(f_{i}\right):\right),
$$

where $\mu_{0}^{c}$ is a 0 -simplicial free measure respectively free measure in continuum defined with $m_{0}>0$.

The corresponding limit result holds for $: e^{\alpha \varphi}:\left(R_{n} f\right)$ as well as for local functions $\vdots \varphi^{N}:$ and $: e^{\alpha \varphi}:$, for all $0 \leqq \alpha \leqq \alpha_{0}$, with $\alpha_{0}$ as in Proposition 4.3. 
Proof. It is sufficient to consider the case when $f_{i}=g, i=1, \ldots, N$, for some $g \in C^{\infty}(M)$ and $f, g \geqq 0, \operatorname{supp} f \cap \partial M=\varnothing$. Let us set

$$
C_{i j} \equiv \mu_{0}^{c}\left(\varphi_{i} \varphi_{j}\right)
$$

Then we have

$$
\begin{aligned}
\mu_{0}^{c}\left(: \varphi^{N}\left(R_{n} f\right)::\left(\varphi\left(R_{n} g\right)\right)^{N}:\right) & =\sum_{i}\left(R_{n} f, \sigma_{i}\right)\left(\sum_{j} C_{i j}\left(\sigma_{j}, R_{n} g\right)\right)^{N} \\
& \equiv \int * W_{n} R_{n} f(x) \sum_{i} W_{n} \sigma_{i}(x)\left(\sum_{j} C_{i j}\left(\sigma_{j}, R_{n} g\right)\right)^{N} .
\end{aligned}
$$

Let us remark that using the identity

and Hölder inequality we get

$$
\sum_{i} W_{n} \sigma_{i}(x) \equiv 1
$$

$$
\begin{aligned}
\sum_{i} W_{n} \sigma_{i}(x)\left(\sum_{j} C_{i j}\left(\sigma_{j}, R_{n} g\right)\right)^{N} & \geqq\left(\sum_{i j} W_{n} \sigma_{i}(x) C_{i j}\left(\sigma_{j}, R_{n} g\right)\right)^{N} \\
& \equiv\left(W_{n} G^{c} R_{n} g(x)\right)^{N} .
\end{aligned}
$$

Therefore

$$
\mu_{0}^{c}\left(: \varphi^{N}:\left(R_{n} f\right):\left(\varphi\left(R_{n} g\right)\right)^{N}:\right) \geqq \int * W_{n} R_{n} f(x)\left(W_{n} G^{c} R_{n} g(x)\right)^{N} .
$$

Since $W_{n} G^{c} R_{n} g \underset{n \rightarrow \infty}{\longrightarrow} G g$ in $H_{+1}$, so it converges (for $D=2$ ) in any $L_{p}, 1<p<\infty$. This implies that for the right-hand side of (5.8) we have

$$
\begin{aligned}
\lim _{n \rightarrow \infty} \int * W_{n} R_{n} f(x)\left(W_{n} G^{c} R_{n} g(x)\right)^{N} & =\int * f(x)(G g(x))^{N} \\
& =\mu_{0}\left(: \varphi^{N}:(f): \varphi(g)^{N}:\right) .
\end{aligned}
$$

To show (5.3) we write (5.5) as follows (recalling the notation $\sigma_{i}=\left[p_{i}\right]$ )

$$
\begin{aligned}
\int * & W_{n} R_{n} f(x) \sum_{i} W_{n} \sigma_{i}(x)\left(W_{n} G^{c} R_{n} g\left(p_{i}\right)\right)^{N} \\
= & \int * W_{n} R_{n} f(x)\left(W_{n} G^{c} R_{n} g(x)\right)^{N} \\
& +\sum_{k=1}^{N}\left(\begin{array}{c}
N \\
k
\end{array}\right) \int * W_{n} R_{n} f(x) \sum_{i} W_{n} \sigma_{i}(x)\left(W_{n} G^{c} R_{n} g\left(p_{i}\right)-W_{n} G^{c} R_{n} g(x)\right)^{k} .
\end{aligned}
$$

By Hölder inequality we get

$$
\begin{aligned}
\mid \int * & W_{n} R_{n} f(x) \sum_{i} W_{n} \sigma_{i}(x)\left(W_{n} G^{c} R_{n} g\left(p_{0}\right)-W_{n} G^{c} R_{n} g(x)\right)^{k} W_{n} G^{c} R_{n} g(x)^{N-k} \mid \\
\leqq & \left(\int * \sum_{i} W_{n} \sigma_{i}(x)\left|W_{n} G^{c} R_{n} g\left(p_{i}\right)-W_{n} G^{c} R_{n} g(x)\right|^{2}\right)^{1 / 2} \\
& \left(\int * \sum_{i} W_{n} \sigma_{i}(x)\left(W_{n} G^{c} R_{n} g\left(p_{i}\right)-W_{n} G^{c} R_{n} g(x)\right)^{4(k-1)}\right)^{1 / 4} \\
& \cdot\left(\int *\left(W_{n} R_{n} f(x)\right)^{4}\left(W_{n} G^{c} R_{n} g(x)\right)^{4(N-k)}\right)^{1 / 4} .
\end{aligned}
$$


The last factor on the right-hand side of (5.11) is uniformly bounded in $n$ (in fact it converges as $n \rightarrow \infty$, by the argument following (5.8), in $L_{p}, p>1$ ). The second factor from the right-hand side of (5.11) can be bounded by using Hölder's inequality and the identity (5.6) by a sum of terms of the form

$$
\left(\int * \sum_{i} W_{n} \sigma_{i}(x)\left(W_{n} G^{c} R_{n} g\left(p_{i}\right)\right)^{N^{\prime}}\right)^{1 / N^{\prime}}\left(\int\left(W_{n} G^{c} R_{n} g(x)\right)^{N^{\prime \prime}}\right)^{1 / N^{\prime \prime}}
$$

with some $N^{\prime}, N^{\prime \prime} \in \mathbb{N}$ dependent only on $N$ and $k$.

We remark

$$
\begin{aligned}
\int * \sum_{i} W_{n} \sigma_{i}(x)\left(W_{n} G^{c} R_{n} g\left(p_{i}\right)\right)^{N^{\prime}} & =\mu_{0}^{c}: \varphi^{N^{\prime}}:(\mathbb{1}):\left(\varphi\left(R_{n} g\right)\right)^{N^{\prime}}: \\
& \leqq\left(\mu_{0}^{c}\left(: \varphi^{N^{\prime}}:(\mathbb{1})^{2}\right)\right)^{1 / 2}\left(\left(\mu_{0}^{c}\left(: \varphi\left(R_{n} g\right)\right)^{N^{\prime}}:\right)^{2}\right)^{1 / 2} .
\end{aligned}
$$

Now using Proposition 4.3 we see that the right-hand side of (5.13) is uniformly bounded as $n \rightarrow \infty$.

Hence to finish our proof we shall have to show that

$$
\lim _{n \rightarrow \infty} \int * \sum_{i} W_{n} \sigma_{i}(x)\left|W_{n} G^{c} R_{n} g\left(p_{i}\right)-W_{n} G^{c} R_{n} g(x)\right|^{2}=0 .
$$

To see this, let us observe that on $D$-simplexes in $K_{n}$ the gradient of $W_{n} G^{c} R_{n} g(x)$ is constant (since there we have a linear function). Therefore we have the estimate (cfr. [StrF])

$$
\int * \sum_{i} W_{n} \sigma_{i}(x)\left|W_{n} G^{c} R_{n} g\left(p_{i}\right)-W_{n} G^{c} R_{n} g(x)\right|^{2} \leqq \eta_{n}^{2} C \int d W_{n} G^{c} R_{n} g \wedge * d W_{n} G^{c} R_{n} g
$$

with a constant $0<C<\infty$ dependent only on the metric. Using the fact that $W_{n} G^{c} R_{n} g$ converges to $G g$ in $H_{+1}$ norm, (5.15) together with (5.10)-(5.14) implies

$$
\int * W_{n} R_{n} f(x) \sum_{i} W_{n} \sigma_{i}(x)\left(W_{n} G^{c} R_{n} g\left(p_{i}\right)\right)^{N} \leqq \int * W_{n} R_{n} f(x)\left(W_{n} G^{c} R_{n} g(x)\right)^{N}+C \eta_{n}^{2},
$$

with some constant $0<C<\infty$ independent of $n$. This together with (5.8) and (5.9) finishes the proof of (5.3).

To show the same statement for $: e^{\alpha \varphi}:\left(R_{n} f\right)$ we use (5.3) together with Proposition 4.3 (inequality (4.7)).

The statements for : : local functions can be proven similarly.

Remark. One can give a simpler proof using the convergence of $W G^{c} R f$ in $\|\cdot\|_{\infty}$ norm, which holds in two dimensions, see [Ni] (on the other hand this convergence requires more complicated considerations than the convergence in $H_{+1}$ ). For another proof of the convergence of the lattice approximation in the case where $M$ is a torus, see [Sc].

Remark. In the discrete approximation of Euclidean fields over $\mathbb{R}^{2}$ where the lattice is taken to be the regular one $\delta \mathbb{Z}^{2}$ it is useful, cfr. [Ne], [GRS], [Si], [GiJ] to realize the discretized fields on the same probability space as for the fields associated with the continuum (this is sometimes called the "active picture"). We remark that we can obtain a similar "continuum representation" for our local functions (4.1) respectively (4.2). 
Let us define, in analogy to the above mentioned procedure, the functions

$$
f_{i}(x) \equiv \sum_{j k} G^{-1 / 2} W \sigma_{j}(W \sigma ., W \sigma .)_{j k}^{-1 / 2} C_{k i}^{1 / 2},
$$

where $i$ is such that $\left(p_{i}\right) \in K$ and $C_{k i}$ is given by (5.4). We also define, in correspondence with the local functions (4.2)

$$
\tilde{f}_{i}(x) \equiv G^{-1 / 2} W\left(G^{c}\right)^{1 / 2} \tilde{\sigma}_{i}
$$

Then by explicit calculation we get

$$
\mu_{0}\left(\varphi\left(f_{i}\right) \varphi\left(f_{j}\right)\right)=C_{i j}
$$

and respectively

$$
\mu_{0}\left(\varphi\left(\tilde{f}_{i}\right) \varphi\left(\tilde{f}_{j}\right)\right)=\left(\tilde{\sigma}_{i} G^{c} \tilde{\sigma}_{j}\right)
$$

This implies that the local functions (4.1) and (4.2) of simplicial fields have the following representation:

$$
: \varphi^{N}:(R f)=\sum_{i}: \varphi\left(f_{i}\right)^{N}:_{\mu_{0}}\left(\sigma_{i}, R f\right)
$$

and respectively

$$
\vdots \varphi^{N}:(R f)=\sum_{i}: \varphi\left(\tilde{f}_{i}\right)^{N}:_{\mu_{0}}\left(\sigma_{i}, R f\right)
$$

Proceeding similarly as in the proof of Proposition 5.1 and of Proposition 4.3 it is not difficult to prove the following:

Proposition 5.2. For any $f \in C^{\infty}(M)$ there is a subsequence $\left\{K_{n^{\prime}}\right\}_{n^{\prime} \in \mathbf{N}}$ such that

$$
\lim _{n^{\prime} \rightarrow \infty} \sum_{i}: \varphi\left(f_{i}\right)^{N}:_{\mu_{0}}\left(\sigma_{i}, R_{n}^{\prime} f\right)=: \varphi^{N}:_{\mu_{0}}(f)
$$

in $L^{p}\left(\mu_{0}\right), 1 \leqq p<\infty$.

The corresponding limit result in $L^{2}\left(\mu_{0}\right)$ holds for the corresponding random variable $: e^{\alpha \varphi}:(R f)$ for all $0 \leqq \alpha<\alpha_{0}$ and some $\alpha_{0}>0$ (independent of the metric) defined with the use of random variables

$$
\left\{\varphi\left(\tilde{f}_{i}\right): \tilde{f}_{i} \equiv G^{-1 / 2} W_{n}\left(G^{c}\right)^{1 / 2} \tilde{\sigma}_{i}\right\}
$$

Corresponding results hold also with the local functions $: \varphi\left(f_{i}\right)^{N}$ : respectively $: e^{\alpha \varphi}:(R f)$ replaced by the corresponding triple dots function $\vdots \varphi\left(\tilde{f}_{i}\right)^{N}:$ respectively $\vdots e^{\alpha \varphi}:(R \widetilde{f})$.

\section{Exponential Model on a Manifold}

Let $\mu_{0}^{c}$ be a free 0 -simplicial measure corresponding to a triangulation $K$ of the manifold $M$, discussed in previous sections. Define the exponential interaction associated with $K$ by

$$
U_{\mathbf{K}}(\varphi):=\sum_{i} \int: e^{\alpha \varphi_{i}}:\left(\sigma_{i}, \mathbb{1}\right) d \rho(\alpha)
$$

with a finite (nonnegative) measure $d \rho(\alpha)$; the summation runs over all $i$ with 
$\sigma_{i} \in C^{0}(K)$ and : : denotes the normal ordering with respect to $\mu_{0}^{c}$, supp $\rho \subset\left(-\alpha_{0}, \alpha_{0}\right)$ (with $\alpha_{0}$ depending on the given metric, as given by Proposition 4.3). To the interaction $U_{K}(\varphi)$ we associate the following probability measure

$$
\mu^{c}(\cdot):=\frac{\mu_{0}^{c}\left(e^{-U_{K} \cdot}\right)}{\mu_{0}^{c}\left(e^{-U_{K}}\right)} .
$$

The essential properties of this measure are described by

Proposition 6.1. For any $f \in C^{\infty}(M)$ and $n \in \mathbb{N}$,

$$
\mu^{c}|\varphi(R f)|^{n} \leqq \mu_{0}^{c}|\varphi(R f)|^{n}
$$

and

$$
0 \leqq \mu^{c}(\varphi(R f), \varphi(R f)) \leqq \mu_{0}^{c}(\varphi(R f))^{2}
$$

as well as

$$
0 \leqq \mu^{c}\left(: \varphi^{2}:(R f),: \varphi^{2}:(R f)\right) \leqq \mu_{0}^{c}\left(: \varphi^{2}:(R f)\right)^{2} .
$$

Proof. Since by definition (6.1) the function $U_{K}(\varphi)$ is convex, (6.3) follows from Lemma 3.2 (based on Brascamp-Lieb's result). To see that (6.4a) holds let us write it in the following form (with $f^{c} \equiv R f$ ):

$$
\mu^{c}\left(\varphi\left(f^{c}\right), \varphi\left(f^{c}\right)\right)=\frac{1}{2} \mu^{c} \otimes \tilde{\mu}^{c}\left(\varphi\left(f^{c}\right)-\tilde{\varphi}\left(f^{c}\right)\right)^{2},
$$

with $\tilde{\mu}^{c}$ an isomorphic copy of $\mu^{c}$. The integration variables on the right-hand side of (6.5), $\varphi$ respectively $\tilde{\varphi}$, correspond to $\mu^{c}$ respectively $\tilde{\mu}^{c}$. Let now change the integration variables, setting

$$
\begin{aligned}
& \xi_{i} \equiv \frac{1}{\sqrt{2}}\left(\varphi_{i}+\tilde{\varphi}_{i}\right) \\
& \eta_{i} \equiv \frac{1}{\sqrt{2}}\left(\varphi_{i}-\tilde{\varphi}_{i}\right) .
\end{aligned}
$$

Using the fact that the measure $\mu_{0}^{c} \otimes \tilde{\mu}_{0}^{c}$ is invariant with respect to this change, we can write (6.5) as follows

$$
\mu^{c}\left(\varphi\left(f^{c}\right), \varphi\left(f^{c}\right)\right)=\frac{1}{Z^{2}} \mu_{0}^{c} \otimes \tilde{\mu}_{0}^{c} e^{-V_{K}(\xi, \eta)}\left(\eta\left(f^{c}\right)\right)^{2},
$$

with $Z$ the normalization factor and

$$
V_{K}(\xi, \eta) \equiv U_{K}\left(\frac{1}{\sqrt{2}}(\xi+\eta)\right)+U_{K}\left(\frac{1}{\sqrt{2}}(\xi-\eta)\right) .
$$

We remark that the conditional expectation of $\eta\left(f^{c}\right)^{2}$ given $\xi$ corresponding to the probability measure $\mu^{c} \otimes \tilde{\mu}^{c}$ is equal to

$$
E_{K}^{\xi}\left(\eta\left(f^{c}\right)^{2}\right) \equiv \frac{\tilde{\mu}_{0}^{c} e^{-V_{K}(\xi, \eta)} \eta\left(f^{c}\right)^{2}}{\tilde{\mu}_{0}^{c} e^{-V_{K}(\xi, \eta)}}
$$

Since $V_{K}(\xi, \eta)$ is a convex function of $\eta$, using again the Brascamp-Lieb inequalities 
(Lemma 3.2) we get

$$
E_{K}^{\xi}\left(\eta\left(f^{c}\right)^{2}\right) \leqq \tilde{\mu}_{0}^{c}\left(\eta\left(f^{c}\right)^{2}\right) .
$$

This together with (6.7) implies (6.4a).

To see that (6.4b) holds we use the same representation and change of integration variables as above. This gives

$$
\mu^{c}\left(: \varphi^{2}:\left(f^{c}\right),: \varphi^{2}:\left(f^{c}\right)\right)=\frac{2}{Z^{2}}\left(\mu_{0}^{c} \otimes \tilde{\mu}_{0}^{c}\right)\left(e^{-V_{K}(\xi, \eta)}\left(\sum_{i} \eta_{i} \xi_{i}\left(\sigma_{i}, f^{c}\right)\right)^{2}\right) .
$$

Applying Lemma 3.2 to the right-hand side of (6.11) we get then (6.4b).

Remark. Using Brascamp-Lieb inequalities one can prove also other inequalities for correlation functions (cf. [Fo], [FröZe]).

Let $\mu_{0}$ be a Gaussian measure with mean zero and covariance

$$
\left(-\Delta+\lambda H+m^{2} I\right)^{-1}
$$

where $\Delta$ is a Laplace-Beltrami operator for functions on the two dimensional Riemannian manifold $M, H$ is the harmonic projection, $\left(\lambda, m^{2}\right) \in\left(\mathbb{R}^{+}\right)^{2}$ (and $\left(\lambda, m^{2}\right) \neq(0,0)$ in case no Dirichlet boundary conditions on $\partial M$ are assumed). Let

$$
U_{M}(\varphi) \equiv \int d \rho(\alpha) \int_{M}: e^{\alpha \varphi}:_{\mu_{0}}(x) \omega_{x}
$$

be the exponential interaction on $M$, where $\omega_{x}$ is the volume element corresponding to a given metric and $d \rho(\alpha)$ is a nonnegative finite measure supported in $\left\{|\alpha|<\alpha_{0}\right\}$ with some $0<\alpha_{0}<\infty$ (independent of the metric) chosen so that $U_{M}(\varphi) \in L^{2}\left(\mu_{0}\right)$ (cf. [AHK], [AHKPS] and above Proposition 4.3). Define a probability measure with exponential interaction in continuum given by

$$
\mu(\cdot):=\frac{\mu_{0}\left(e^{-U_{M}(\varphi)}\right)}{\mu_{0}\left(e^{-U_{M}(\varphi)}\right)} .
$$

One can easily see, using properties of $U_{M}(\varphi)$, that $\mu$ is well defined, at least when $M$ is compact, cf. [AHK], [AHKPS]. In the case where $M$ is non-compact one can construct $\mu(\cdot)$ as a weak subsequences limit of

$$
\frac{\mu_{0}\left(e^{-U_{M}^{A}(\varphi)}\right)}{\mu_{0}\left(e^{-U_{M}^{A}(\varphi)}\right)}
$$

as $\Lambda \uparrow M$, with $\Lambda$ compact, by using Brascamp-Lieb inequalities and by going through subsequences.

Theorem 6.2. The measure $\mu$ with exponential interaction on a Riemannian manifold defined in (6.14) is the limit (in the sense of characteristic functionals and convergence of moments) of the probability measures $\mu^{c}$ as the mesh $\eta$ converges to zero. $\mu$ satisfies

$$
\mu \underset{B-L}{\varrho} \mu_{0}
$$

i.e. (3.4) holds for the measures considered here. Moreover $(6.4 a, b)$ holds with $\mu^{c}$ respectively $\mu_{0}^{c}$ replaced by $\mu$ respectively $\mu_{0}$. 
Proof. The proof is constructed following the model of the proof of correlation inequalities in the flat space, of [AHK]. First one discusses the case $m^{2}>0$, using the convergence of the lattice approximation given in Sect. 5, which yields $\mu$ as limit for $\eta \downarrow 0$ of the probability measures $\mu^{c}$ (in the sense of characteristic functionals and convergence of moments). Moreover one uses Proposition 6.1. The continuity of expectations in $m$ then yields the proof for the case $\lambda>0$ and $m^{2}=0$.

Remark. One can get the same result directly in the continuum limit, for $M$ compact (or $M$ replaced by a compact subset of it) using the decomposition of $\mu_{0}$ into orthogonal modes and a finite modes approximation for the interaction $U_{M}(\varphi)$.

Remark. Let us note that use of Brascamp-Lieb inequalities allows to considerably simplify the proofs (given in [Ze]) of extremality and global Markov property for euclidean field with a general exponential interaction in two dimensions. (For other applications of these inequalities see e.g. [BraLie], [BriFLS], [Fo], [FröZe].)

Remark. We expect that the above convergence of simplicial approximation implies also GKS and FKG inequalities if we take $\lambda_{0}=0$. To prove this we shall take a local mass term

$$
\frac{m^{2}}{2} \sum \varphi_{i}^{2}\left(\sigma_{i}, \mathbb{1}\right)
$$

instead of (nonlocal, antiferromagnetic) term

$$
\frac{m^{2}}{2} \sum \varphi_{i} \varphi_{j}\left(\sigma_{i}, \sigma_{j}\right)
$$

and choose an interaction given by a : - Wick ordered local functions. The proof depends in a critical way on a choice of "normal" triangulation corresponding to a choice of normal coordinates associated to a given metric. (As we saw in Example 3.5, for a given metric $\left\{g^{\mu \nu}\right\}$, an arbitrary choice of triangulation can spoil ferromagneticity.) It is easy to get a proof of mentioned correlation inequalities for canonical metrics
$d$-dimensional torus. $\left|\sum_{i=1}^{d} \tau_{i} d x^{i}\right|^{2}$, defined with some constants $\tau_{i} \in \mathbb{C}$, in case of the

We postpone a study of the general case to future work.

Acknowledgements. We are very grateful to Zbigniev Haba, Erling Heyerdahl-Hohler, Sylvie Paycha and Sergio Scarlatti for very interesting and stimulating discussions. We thank Martin Jarrath for the expert setting of the manuscript. We would like also to thank Józef Dodziuk for his interest and useful remarks.

The financial support of the SFB 237 Bochum-Essen-Düsseldorf is also gratefully acknowledged.

\section{References}

[A] Albeverio, S.: Some new developments concerning Dirichlet forms, Markov fields and quantum fields, pp. 250-259. In: Mathematical Physics. Swansea '88. Simon, B., Truman, A., Davies, I. M. (eds.) Bristol: Adam Hilger 1988

[AFHKL] Albeverio, S., Fenstad, J. E.: Høegh-Krohn, R., Lindstrøm, T.: Non-standard methods in stochastic analysis and mathematical physics. Orlando: Academic Press 1986 
[AHK] Albeverio, S., Høegh-Krohn, R.: The Wightman axioms and the mass gap for strong interactions of exponential type in two-dimensional space time. J. Funct. Anal. 16, 39-82 (1974)

[AHKH] Albeverio, S., Høegh-Krohn, R., Holden, H.: Markov cosurfaces and gauge fields. Acta Phys. Austr. [Supl.] XXVI, 211-231 (1984)

[AHKHK1] Albeverio, S., Høegh-Krohn, R., Holden, H., Kolsrud, T.: Stochastic multiplicative measures, generalized Markov semigroups and group valued stochastic processes and fields. J. Funct. Anal. 78, 154-184 (1988)

[AHKHK2] Albeverio, S., Høegh-Krohn, R., Holden, H., Kolsrud, T.: Representation and construction of multiplicative noise, J. Funct. Anal. 87, 250-272

[AHKHK3] Albeverio, S., Høegh-Krohn, R., Holden, H., Kolsrud, T.: Construction of quantized Higgs-like fields in two dimensions. Physics Letters B 222, 263-268 (1989)

[AHKI] Albeverio, S., Høegh-Krohn, R., Iwata, K.: Covariant Markovian random fields in four space-time dimensions with nonlinear electromagnetic interaction, pp. 69-83. Exner, P., Seba, P. (eds.), Lecture Notes Physics, vol. 324. Berlin, Heidelberg, New York: Springer 1989

[AHKMTT] Albeverio, S., Høegh-Krohn, R., Marion, J., Testard, D., Torresani, B.: Non-commutative distribution theory, book in preparation

[AHKPS1] Albeverio, S., Høegh-Krohn, R., Paycha, S., Scarlatti, S.: A probability measures for random surfaces of arbitrary genus and bosonic strings in 4 dimensions. Nucl. Phys. $B$ (Proc. Suppl.) 6, 180-182 (1989)

[AHKPS2] Albeverio, S., Høegh-Krohn, R., Paycha, S., Scarlatti, S.: A global and stochastic analysis approach to bosonic strings and associated quantum fields. Bochum Preprint (1989)

[AHPRS] Albeverio, S., Hida, T., Potthoff, J. Röckner, M., Streit, L.: Dirichlet forms in terms of white noise analysis. I. Construction of QFT examples; II. Construction of infinite dimensional diffusions. BiBoS Preprint (1989), to appear in Rev. Math. Phys. (1990)

[AIK] Albeverio, S., Iwata, K., Kolsrud, T.: Random fields as solutions of the inhomogeneous quaternionic Cauchy-Riemann equation. I. Invariance and analytic continuation. Bochum Preprint (1989), to appear in Commun. Math. Phys. (1990)

[AK] Albeverio, S., Kusuoka, S.: Maximality of infinite dimensional Dirichlet forms and R. Høegh-Krohn's model of quantum fields. Memorial Volume for Raphael Høegh-Krohn (to appear)

[AR1] Albeverio, S., Röckner, M.: Classical Dirichlet forms on topological vector spaces-The construction of the associated diffusion process. Prob. Th. Rel. Fields 83, 405-434 (1989)

[AR2] Albeverio, S., Röckner, M.: New developments in theory and applications of Dirichlet forms, to appear in Stochastic Processes-Geometry and Physics, Proc. AsconaLocarno-Como, Singapore: World Scientific 1989

[Ad] Adler, R. J.: The geometry of random fields. Chichester: J. Wiley 1981

[AmD] Ambjørn, J., Durhuus, B.: Regularized bosonic strings need extrinsic curvature. Phys. Letts. 188B, 253-257 (1987)

[As] Ashtekar, A: Recent developments in Hamiltonian gravity, pp. 268-271. In: Mathematical Physics. Simon, B., Truman, A., Davies, I. M. (eds.). Bristol: Adam Hilger 1989

[BaJ] Balaban, T., Jaffe, A.: Constructive gauge theory, pp. 207-263. In: Velo, G., Wightman, A. S. (eds.) Fundamental Problems of Gauge Field Theory. Amsterdam: D. Reidel 1986

[BraLie] Brascamp, H. J., Lieb: E. H.: On Extensions of the Brunn-Minkowski and PrékopaLeindler Theorems, including Inequalities for Log Concave Functions, and with an Applications to the Diffusion Equation. J. Funct. Anal. 22, 366-389 (1976)

[BriFLS] Bricmont, J., Fontaine, J. R., Lebowitz, J. L., Spencer, T.: Lattice systems with a continuous symmetry; Part I. Commun. Math. Phys. 78, 281-302 (1980); Part II. Commun. Math. Phys. 78, 263-372 (1981); Part III with Lieb, E.: Commun. Math. Phys. 78, 545-566(1981)

[CDeA] Dell' Antonio, G. F., Cotta-Ramusino, P.: Self duality and topologial-like properties of lattice gauge field theories. A proposal. Commun. Math. Phys. 70, 75-95 (1979)

[CMS] Cheeger, J., Müller, W., Schrader, R.: On the curvature of piecewise flat spaces. Commun. Math. Phys. 92, 405-454 (1984)

[Che] Cheeger, J.: Analytic torsion and the heat equation. Ann. Math. 109, 259-322 (1979) 
[Cia] Ciarlet, Ph.: Lectures on The Finite Element Method. Tata Institute of Fundamental Research, Bombay, India 1975

[DeADFG] De Angelis, G. E., De Falco, D., Guerra, F.: Scalar quantum electrodynamics on the lattice as classical statistical mechanics. Commun. Math. Phys. 57, 201-212 (1977)

[Do] Dodziuk, J.: Finite-Difference approach to the Hodge Theory of Harmonic Forms. Am. J. Math. 98, 79-104 (1976)

[DoP] Dodziuk, J., Patodi, V. K.: Riemannian structures and triangulations of manifolds. J. Indian Math. Soc. 40, 1-52 (1976)

[Dr] Driver, B. K.: Convergence of the $U(1)_{4}$ lattice gauge theory to its continuum limit. Commun. Math. Phys. 110, 479-501 (1987)

[DrM] Drouffe, J. M., Moriarty, K. J. M.: Gauge theories on a simplicial lattice. Nucl. Phys. B 220, 253-268 (1983)

[Eck] Eckman, B.: Harmonische Funktionen and Randwertaugfaben in einem Komplex. Commentari Math. Helv. 17, 240-245 (1944/45)

[El] Elworthy, D.: Lectures on stochastic differential equations, St. Flour (1988)

[EIR] Elworthy, K. D., Rosenberg, S.: Spectral bounds and the shape of manifolds near infinity, pp. 369-373. In: Mathematical Physics. Swansea '88. (eds.). Simon, B., Truman, A., Davies, I. M. Bristol: Adam Hilger 1989

[FKG] Fortuin, C. M., Kastelyn, P. W.., Ginibre, J.: Correlation inequalities on some partially ordered sets. Commun. Math. Phys. 22, 89-103 (1971)

[FreLM] Frenkel, I., Lepowsky, J., Meurman, A.: Vertex operator algebras and the monster. Boston: Academic Press 1988

[Fo] Fontaine, J. R.: Bounds on the Decay of Correlations for $\lambda(\nabla \Phi)^{4}$ Models. Commun. Maths. 87, 835-394 (1982)

[Frö] Fröhlich, J.: Statistics of fields, the Yang-Baxter equation and the theory of knots and links. t' Hooft, G. et al. (eds.). New York: Plenum Press 1988

[FröZe] Fröhlich, J., Zegarlinski, B.: Spin Glasses and other Lattice Systems with Long Range Interactions. Commun. Math. Phys. 120, 665-668 (1989)

[Ga] Gawedzki, K.: Conformal field theory. Sém. Bourbaki 41e année, 1988-89, No. 704

[GIJ] Glimm, J., Jaffe, A.: Quantum physics: A functional integral point of view. Second ed, Berlin, Heidelberg New York: Springer 1987

[GRS] Guerra, F., Rosen, L., Simon, B.: The $P(\varphi)_{2}$ Euclidean quantum field theory as classical statistical Mechanics. Ann. Math. 101, 111-259 (1975)

[GSW] Green, M. B., Schwartz, J. H., Witten, E.: Superstring theory. Cambridge University Press 1982

[Gi] Ginibre, J.: General formulation of Griffith's inequalities. Commun. Math. Phys. 16, 310 (1970)

[Gr1] Gross, L.: Logarithmic Sobolev inequalities. Am. J. Math. 97, 1061-1083 (1976)

[Gr2] Gross, L.: Convergence of $U(1)_{3}$ lattice gauge theory to its continuum limit. Commun. Math. Phys. 92, 137-162 (1983)

[GrKS] Gross, L., King, Ch., Sengupta, A.: Two dimensional Yang-Mills theory via stochastic differential equations. Cornell University Preprint (1989)

[Gri] Griffiths, R.: Correlations in Ising Ferromagnets I, II, III. J. Math. Phys. B (1967) 478-483, 484-489; Commun. Math. Phys. 6, 121-127 (1967)

[HaI] Hawking, S. W., Israel, W. eds.: Three hundred years of gravitation (1987)

[I] Ito, K.: Isotropic random currents, pp. 125-132 in Proc. 3 d Berkeley Sympos. Math. Sta. Prob. (1956)

[ISZ] Itzykson, C., Saleur, H., Zuber, J. B. eds.: Conformal invariance and applications to statistical mechanics. Singapore: World Scientific 1988

[KS] Kelly, D., Sherman, S.: General Griffith's inequalities on Correlations on Ising Ferromagnets. J. Math. Phys. 9, 466-488 (1968)

[Kac] Kac, V.: Infinite dimensional Lie algebras. Boston: Birkhäuser 1983

[Kau] Kaufmann, L. H.: Knot polynomials and Yang-Baxter models, pp. 438-441. In: Mathematical Physics. Swansea 88. Simon, B., Trumann, A., Davies, I. M. (eds.). Briston: Adam Hilger 1989 
[Kha] Khatsymovsky, V. M.: Vector fields and gravity on the lattice. Novosibirsk Preprint (1988)

[Kr] Krylov, A. L.: Difference approximations to differential operators of mathematical physics. Sov. Math. Dokl. 9, 138-141 (1968)

[Ku] Kusuoka, S.: Høegh-Krohn's model of quantum fields and the absolute continuity of measures. To appear in Memorial Volume for R. Høegh-Krohn

[Le] Leff, H. S.: Correlation Inequalities for Coupled Oscillators. J. Math. Phys. 1, 569-578 (1971)

[Ma] Mandelbrot, B. B.: The fractal geometry of nature. New York: W. H. Freeman 1983

[MBF] Markov, M. A., Berezin, V. A., Frolov, V. P.: Quantum Gravity, Moscow 1987, Singapore: World Scientific 1988

[Mü] Müller, W.: Analytic Torsion and R-Torsion of Riemannian Manifolds. Adv. Math. 28, 233-305 (1978)

[Ne] Nelson, E.: The free Markoff field. J. Funct. Anal. 12, 211-227 (1973)

[Nit] Nitsche, J.: $L_{\infty}$-convergence of finite element approximations, pp. 261-274. In: Mathematical Aspects of Finite Element Methods. Galligani, I., Magenes, E. (eds.) Lecture Notes in Mathematics, vol. 606. Berlin, Heidelberg, New York: Springer 1977, see also ref. [12] there

[PrS] Pressley, A., Segal, G.: Loop groups. Oxford: Oxford University Press 1986

[RayS] Ray, D. B., Singer, L. M.: R-Torsion and the Laplacian on Riemannian Manifolds. Adv. Math. 7, 145-210 (1971)

[Re] Regge, T.: General relativity without coordinates. Nuovo Cim. 10, 558-571 (1961)

[RehS] Rehren, K. H., Schroer, B.: Einstein causality and Artin braids, FU Preprint (1988)

[Sa] Santaló, L. A.: Integral geometry and geometric probability. Enc. Maths. Appl., Vol. 1, Reading, MA: Addison-Wesley 1986

[Sc] Scarlatti, S.: PhD Thesis, Roma, 1989

[Si] Simon: B. The $P(\varphi)_{2}$ Euclidean (Quantum) Field Theory. Princeton, NJ: Princeton University Press 1974

[SiTh] Singer, I. M., Thorpe, J. A.: Lecture notes on elementary topology and geometry. Berlin, Heidelberg, New York: Springer 1967

[StrF] Strang, G., Fix, G. J.: An analysis of the finite element method. Englewood Cliffs, NJ: Prentice-Hall 1973

[Wa] Warner, F.W.: Foundations of differentiable manifolds and Lie groups. Glenview: Scott, Foresman and Company 1971

[Whi] Whitney, H.: Geometric Integration Theory. Princeton NJ: Princeton University Press 1957

[Wi] Williams, D.: Diffusions, Markov Processes and Martingales. Chichester: Wiley 1979

[WoZ] Wong, E., Zakai, M.: Isotropic Gauss-Markov currents. Prob. Theory Rel. Fields 82, 137-154 (1989)

[Ze] Zegarlinski, B.: Uniqueness and the Global Markov Property for Euclidean fields: The case of general exponential interaction. Commun. Math. Phys. 96, 195-221 (1984)

Communicated by K. Gawedzki

Received July 30, 1989 
